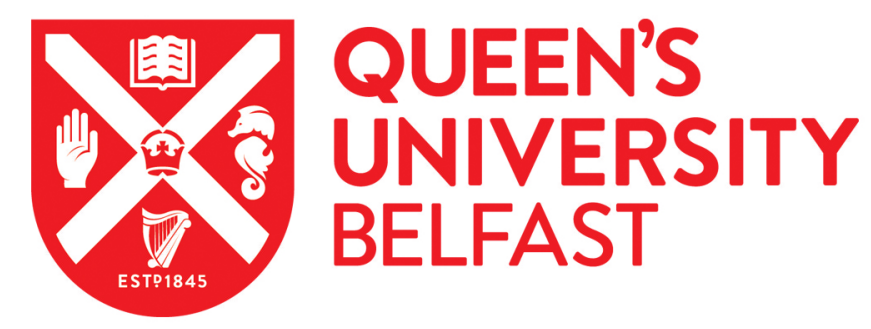

\title{
Observations of SN 2017ein Reveal Shock Breakout Emission and A Massive Progenitor Star for a Type Ic Supernova
}

Xiang, D., Wang, X., Mo, J., Wang, L., Smartt, S., Fraser, M., Ehgamberdiev, S. A., Mirzaqulov, D., Zhang, J., Zhang, T., Vinko, J., Wheeler, J. C., Hosseinzadeh, G., Howell, D. A., McCully, C., DerKacy, J. M., Baron, E., Brown, P., Bi, S., ... Blinnikov, S. (2019). Observations of SN 2017ein Reveal Shock Breakout Emission and A Massive Progenitor Star for a Type Ic Supernova. The Astrophysical Journal. https://doi.org/10.3847/15384357/aaf8b0

Published in:

The Astrophysical Journal

Document Version:

Peer reviewed version

Queen's University Belfast - Research Portal:

Link to publication record in Queen's University Belfast Research Portal

\footnotetext{
Publisher rights

(C) 2019. The American Astronomical Society. All rights reserved.This work is made available online in accordance with the publisher's policies. Please refer to any applicable terms of use of the publisher.
}

\section{General rights}

Copyright for the publications made accessible via the Queen's University Belfast Research Portal is retained by the author(s) and / or other copyright owners and it is a condition of accessing these publications that users recognise and abide by the legal requirements associated with these rights.

Take down policy

The Research Portal is Queen's institutional repository that provides access to Queen's research output. Every effort has been made to ensure that content in the Research Portal does not infringe any person's rights, or applicable UK laws. If you discover content in the Research Portal that you believe breaches copyright or violates any law, please contact openaccess@qub.ac.uk. 
Draft version December 10, 2018

Typeset using LATEX twocolumn style in AASTeX62

Observations of SN 2017ein Reveal Shock Breakout Emission and A Massive Progenitor Star for a Type Ic Supernova

Danfeng Xiang, ${ }^{1}$ Xiaofeng Wang,${ }^{1}$ Jun Mo, ${ }^{1}$ Linguun Wang, ${ }^{2}$ Stephen Smartt,${ }^{3}$ Morgan Fraser ${ }^{4}$ Shuhrat A. Ehgamberdiev, ${ }^{5}$ Davron Mirzaqulov, ${ }^{5}$ Jujia Zhang, ${ }^{6,}, 7,8$ Tianmeng Zhang, ${ }^{9}$ Jozsef Vinko, ${ }^{10,} 11,12$ J. Craig Wheeler, ${ }^{12}$ Griffin Hosseinzadeh, ${ }^{13}, 14$ D. Andrew Howell, ${ }^{13}, 14$ Curtis McCully, ${ }^{13}$ James M DerKacy, ${ }^{15}$ E. Baron, ${ }^{15}$ Peter Brown, ${ }^{16}$ Xianfei Zhang, ${ }^{17}$ Shaolan Bi, ${ }^{17}$ Hao Song, ${ }^{1}$ Kaicheng Zhang, ${ }^{1}$ A. Rest, ${ }^{18,19}$ Ken'ichi Nomoto, ${ }^{20}$ Alexey Tolstov, ${ }^{20}$ And Sergei Blinnikov $21,22,20$

${ }^{1}$ Physics Department and Tsinghua Center for Astrophysics (THCA), Tsinghua University, Beijing, 100084, China

${ }^{2}$ Astroparticle Physics, Institute of High Energy Physics, Chinese Academy of Sciences, Beijing 100049, China

${ }^{3}$ Astrophysics Research Centre, School of Mathematics and Physics, Queens University Belfast, Belfast BT7 1NN, UK

${ }^{4}$ School of Physics, University College Dublin, Belfield, Dublin 4, Ireland

${ }^{5}$ Ulugh Beg Astronomical Institute, Uzbekistan Academy of Sciences, Uzbekistan, Tashkent, 100052, Uzbekistan

${ }^{6}$ Yunnan Observatories, Chinese Academy of Sciences, Kunming 650216, China

${ }^{7}$ Key Laboratory for the Structure and Evolution of Celestial Objects, Chinese Academy of Sciences, Kunming 650216, China

${ }^{8}$ Center for Astronomical Mega-Science, Chinese Academy of Sciences, 20A Datun Road, Chaoyang District, Beijing, 100012, China

${ }^{9}$ National Astronomical Observatory of China, Chinese Academy of Sciences, Beijing, 100012, China

${ }^{10}$ Konkoly Observatory, MTA CSFK, Konkoly Thege M. ut 15-17, Budapest, 1121, Hungary

${ }^{11}$ Department of Optics \&5 Quantum Electronics, University of Szeged, Dom ter 9, Szeged, 6720 Hungary

${ }^{12}$ Department of Astronomy, University of Texas at Austin, Austin, TX, 78712, USA

${ }^{13}$ Las Cumbres Observatory, 6740 Cortona Drive, Suite 102, Goleta, CA 93117-5575, USA

${ }^{14}$ Department of Physics, University of California, Santa Barbara, CA 93106-9530, USA

${ }^{15}$ Homer L. Dodge Department of Physics and Astronomy, University of Oklahoma, Norman, OK

${ }^{16}$ George P. and CynthiaWoods Mitchell Institute for Fundamental Physics \& Astronomy, Texas A. ES M. University, Department of Physics and Astronomy, 4242 TAMU, College Station, TX 77843, USA

${ }^{17}$ Department of Astronomy, Beijing Normal University, Beijing 100875, China

${ }^{18}$ Space Telescope Science Institute, 3700 San Martin Drive, Baltimore, MD 21218, USA

${ }^{19}$ Department of Physics and Astronomy, Johns Hopkins University, Baltimore, MD 21218, USA

${ }^{20}$ Kavli Institute for the Physics and Mathematics of the Universe (WPI), The University of Tokyo Institutes for Advanced Study, The University of Tokyo, 5-1-5 Kashiwanoha, Kashiwa, Chiba 277-8583, Japan

${ }^{21}$ Institute for Theoretical and Experimental Physics (ITEP), 117218, Moscow, Russia

${ }^{22}$ All-Russia Research Institute of Automatics (VNIIA), 127055, Moscow, Russia

\section{ABSTRACT}

We present optical and ultraviolet observations of nearby type Ic supernova SN 2017ein as well as detailed analysis of its progenitor properties from both the early-time observations and the prediscovery Hubble Space Telescope (HST) images. The optical light curves started from within one day to $\sim 275$ days after explosion, and optical spectra range from $\sim 2$ days to $\sim 90$ days after explosion. Compared to other normal SNe Ic like SN 2007gr and SN 2013ge, SN 2017ein seems to have more prominent CII absorption and higher expansion velocities in early phases, suggestive of relatively lower ejecta mass. The earliest photometry obtained for SN 2017ein show indications of shock cooling. The best-fit obtained by including a shock cooling component gives an estimate of the envelope mass as $\sim 0.02 \mathrm{M}_{\odot}$ and stellar radius as $8 \pm 4 \mathrm{R}_{\odot}$. Examining the pre-explosion images taken with the HST WFPC2, we find that the SN position coincides with a luminous and blue point-like source, with an extinctioncorrected absolute magnitude of $\mathrm{M}_{V} \sim-8.2 \mathrm{mag}$ and $\mathrm{M}_{I} \sim-7.7 \mathrm{mag}$. Comparisons of the observations to the theoretical models indicate that the counterpart source was either a single WR star or a binary with whose members had high initial masses, or a young compact star cluster. To further distinguish between different scenarios requires revisiting the site of the progenitor with HST after the SN fades away.

Keywords: supernova: general - supernova: individual: SN 2017ein

\section{INTRODUCTION}

Observationally, type Ic supernovae (SNe Ic) represent a subclass of core-collapse $\mathrm{SNe}(\mathrm{CCSNe})$ that do not 
show either hydrogen or helium features in their spectra. These events, together with $\mathrm{SNe} \mathrm{IIb}$ and $\mathrm{Ib}$, are all called stripped-envelope (SE) SNe (Clocchiatti et al. 1996) due to the fact that all or part of the outer $\mathrm{H} / \mathrm{He}$ envelopes have been stripped from their progenitors prior to explosion. The absence of helium absorptions in the spectra further distinguishes $\mathrm{SNe}$ Ic from SNe Ib. However, recent studies suggest that residual helium seems to exist in the former subclass, e.g., SN 2007gr (Chen et al. 2014), SN 2016coi (Prentice et al. 2018; Yamanaka et al. 2017), and recent statistical analysis from Fremling et al. (2018), suggestive of a continuous distribution between SNe Ic and SNe Ib. Modeling by Dessart et al. (2012) indicates that nickel mixing and hidden He during the explosion can make differences to the appearance of $\mathrm{He}$ in SNe spectra, and progenitors with similar structures can produce both type Ib and Ic. While there are also some studies arguing for little or no helium in SNe Ic (Frey et al. 2013; Liu et al. 2016). Classification of SE-SNe is revisited in Prentice \& Mazzali (2017) based on the strength of He lines in the SNe spectra.

Among SNe Ic, a subgroup called broad-lined supernovae (SNe Ic-BL) gained more attention due to their broad spectral line profiles, relativistic ejecta velocities, and higher luminosities. Some SNe Ic-BL are found to be associated with long gamma-ray bursts (LGRBs), called GRB-SNe, e.g., SN 1998bw (Galama et al. 1998) and SN 2002ap (Gal-Yam et al. 2002). And those that are not associated with GRBs are thought to be due to an artifact of viewing angle, as GRB radiation is strongly beamed. While late-time radio observations of some SNe Ic-BL, e.g. SN 2002ap (Berger et al. 2002), SN 2010ah (Corsi et al. 2011), exclude relativistic jets accompanying these supernovae. Consequently, not all SNe Ic-BL that not associated with GRB are due to beaming effects. Nevertheless, progenitors of these two subclasses of SNe Ic-BL are found to differ in metallicity (Modjaz et al. 2011, 2008). For instance, GRB-SNe occur preferentially in metal-poor galaxies relative to $\mathrm{SNe}$ Ic-BL and other SNe Ic. These facts demonstrate that SNe Ic-BL may have different origins and/or explosion mechanisms compared with normal SNe Ic. Moreover, GRB-SNe may be used as standard candles to measure cosmic distances and cosmological parameters (Li et al. 2014; Cano 2014).

The progenitors of $\mathrm{SNe} \mathrm{Ib} / \mathrm{c}$ have been thought to be Wolf-Rayet (WR) stars with high initial masses $\left(\mathrm{M}_{\mathrm{ZAMS}} \gtrsim 25 \mathrm{M}_{\odot}\right)$ (Crowther 2007). Before corecollapse, these stars usually have experienced severe mass loss through strong stellar winds or due to interaction with companion stars (Paxton et al. 2015; van der Hucht 2006). As the evolution of massive stars is usually dominated by binary evolution (Heger et al. 2003), and also depends largely on metallicity and rotation etc. (Georgy et al. 2013; Georgy et al. 2012; Heger et al. 2003), this makes the direct identification of their progenitors complicated (Smartt 2015). However, there are increasing studies suggesting that a lower mass binary scenario is more favorable for most SNe $\mathrm{Ib} / \mathrm{c}$, considering the measured low ejecta masses (Eldridge et al. 2013; Lyman et al. 2016). And the $\mathrm{H} / \mathrm{He}$ envelopes of the progenitor stars are stripped by binary interaction. There are many detections of progenitor stars for SNe II. For example, most SNe IIP are found to originate from red supergiants (Smartt et al. 2009), while SNe IIL are typically from progenitors with somewhat warmer colors (see Smartt 2015, for a review), and SNe IIb are from those with higher effective temperatures such as yellow supergiants which have had their $\mathrm{H} / \mathrm{He}$ envelopes partially stripped through binary interaction (e.g. SN 1993J; Fox et al. 2014; Maund et al. 2004; Podsiadlowski et al. 1993). Until recently, there has been only one report of the possible identification of progenitor star for SNe Ib, namely iPTF 13bvn, which was proposed to spatially coincide with a single WRlike star identified on the pre-explosion Hubble Space Telescope (HST) images (Groh et al. 2013; Cao et al. 2013). But such an identification is still controversial (e.g. Bersten et al. 2014; Eldridge \& Maund 2016; Eldridge et al. 2015; Fremling et al. 2014). Direct detection of progenitor stars is still elusive for SNe Ic, which prevents us from further testing the theoretical evolution of massive stars (Eldridge et al. 2013).

During the core collapse explosions of massive stars, a "breakout" may occur when the shock breaks through the stellar surface or circumstellar medium (CSM). This process should be accompanied by an X-ray and/or UV flash on a timescale of a few minutes to $1-2$ hours due to high temperatures and is then followed by UV/optical "cooling" emission from the expanding envelope ${ }^{1}$, where both the duration and strength of the cooling depend on the size and density of the stellar envelope or the CSM (Waxman \& Katz 2016). Thus, the shock breakout and the cooling observations can provide information on the progenitor stars or circumstellar environment. Owing to

\footnotetext{
1 The word "envelope" is usually used to represent the $\mathrm{H}$ or $\mathrm{He}$ layers of a star during its evolution. While in shock breakout theory, "envelope" is used as an indication of a thin layer of mass in which the shock breaks out. In this paper, to distinguish between the two interpretations, we use the following rules: "envelope" appears together with " $\mathrm{H}$ " or "He" for the former case; while the others for the latter case. In particular, "stellar envelope" represents the envelope at the surface of the star.
} 
a very short timescale, the shock breakout phenomenon has been possibly detected for very few events, including type Ib SN 2008D (Modjaz et al. 2009; Mazzali et al. 2008), the GRB-SN SN 2006aj (Campana et al. 2006; Waxman et al. 2007), and type IIb SN 2016gkg (Bersten et al. 2018). By comparison, the cooling phase can last for a few days and can be more easily captured, e.g. Arcavi et al. (2017) for SN 2016gkg. For normal SNe Ic, the shock breakout cooling might have been observed in only one object, i.e., LSQ14efd (Barbarino et al. 2017).

SN 2017ein provides us a rare opportunity to constrain the progenitors of $\mathrm{SNe} \mathrm{Ib} / \mathrm{c}$, as it has both the pre-discovery HST images and very early-time photometry obtained within a fraction of a day after explosion. SN 2017ein was discovered in NGC 3938 by Ron Arbour on 2017 May 25.97 (UT dates are used throughout this paper), with a magnitude of $17.6 \mathrm{mag}$ in a clear filter. NGC 3938 is a very nearby type Sc, face-on spiral galaxy at a distance of $\sim 17 \mathrm{Mpc}$, which has produced SN 1961U (type II), SN 1964L (type Ic), and SN 2005ay (type II) before SN 2017ein. Figure 1 shows the finder chart of SN 2017ein which has coordinates of R.A. $=11 \mathrm{~h} 52 \mathrm{~m} 53 \mathrm{~s} .25$, Decl. $=+44^{\circ} 07^{\prime} 26^{\prime \prime} .20$ (J2000). A spectrum was obtained for SN 2017ein half a day after its discovery, and it was classified as a type Ic supernova near maximum light or one that suffered significant reddening (Xiang et al. 2017). Follow-up photometry indicated that this supernova showed a rapid rise in the brightness, revealing that it was discovered at a very young phase (Im et al. 2017). Moreover, the prediscovery photometric data extracted from the Asteroid Terrestrial-impact Last Alert System (ATLAS; Tonry et al. 2018) survey images indicate that the observations of SN 2017ein can be traced back to a phase within a fraction of one day after explosion, representing one of the earliest detection for a type Ic supernova.

In this paper we present extensive optical and ultraviolet (UV) observations of SN 2017ein, including light curves covering from $\sim 1-275$ days after explosion and spectra covering from $\sim 1.8-90$ days. These datasets are used to constrain the explosion parameters and determine the properties of the progenitor star for a normal SN Ic. Moreover, we also analyze the prediscovery HST images to further constrain the progenitor star of SN 2017ein. The paper is structured as follows. In section 2, our photometric and spectroscopic observations of SN 2017ein are presented. In section 3, the light curves are described and analyzed. In section 4 we discuss the distance, foreground extinction to the SN and the absolute magnitudes of SN 2017ein. The spectral evolution of SN 2017ein is presented in section 5 . We model the light curves to place constraints on the progenitor in section 6 . In section 7, we perform the analysis of the properties of stars at and near the SN position on the pre-discovery HST images. Discussions of our results and conclusions are given in section 8 .

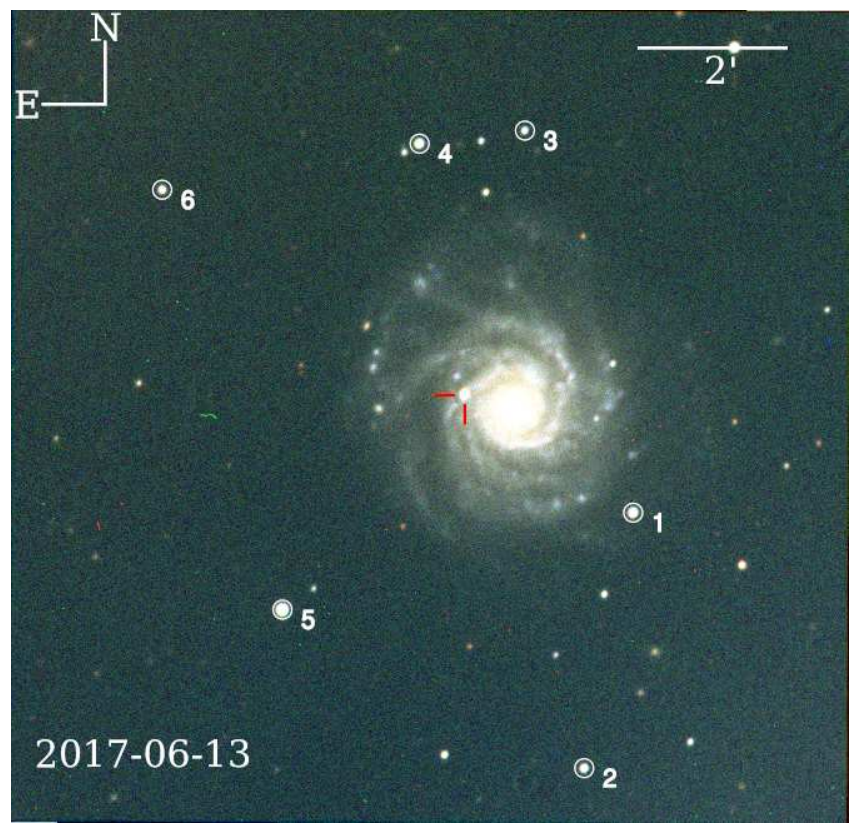

Figure 1. A pseudo-color finder chart of SN 2017ein and its host galaxy NGC 3938, produced from the BVRI-band images obtained with the Tsinghua-NAOC 0.8-m telescope on 2017 June 13. SN 2017ein is marked by red ticks. The comparison stars used for flux calibration are marked by white circles. North is up and west is right.

\section{OBSERVATIONS AND DATA REDUCTION}

As SN 2017ein was discovered at a young phase and it is located at a relatively close distance, we triggered followup observations immediately after its discovery. This supernova was observed in both optical and ultraviolet (UV) bands with the Swift-UVOT (Roming et al. 2005; Gehrels et al. 2004).

\subsection{Optical and ultraviolet photometric obervations}

Photometric observations of SN 2017ein were collected with several telescopes, including the $0.8-\mathrm{m}$ Tsinghua University-NAOC telescope (TNT, Huang et al. 2012) at Xinglong Observatory of NAOC, the AZT-22 1.5-m telescope (hereafter AZT) at Maidanak Astronomical Observatory (Ehgamberdiev 2018). The TNT and AZT observations were obtained in standard Johnson-Cousin BVRI bands. These observations covered the phases from MJD 57904 (2017 May 31) to MJD 58170 (2018 Feb. 22). At late times, the supernova is only visible on the $R$ - and $I$-band images on MJD 58107 (2017 Dec. 20) and becomes undetectable after that. 
ATLAS is a twin 0.5-m telescope system that surveys in cyan (c) and orange (o) filters (Tonry et al. 2018). The typical sky coverage is once every 2 nights. ATLAS monitored the field of SN 2017ein from 2017 Apr. 26, and it did not detect any flux excess relative to the background until 2017 May 25.33 when the SN became detectable at an $\mathrm{AB}$ magnitude of $18.43 \pm 0.10 \mathrm{mag}$ in the $c$-band, which is about 0.64 day before the first discovery. The SN was also detectable in the $o$-band image taken on 2017 May 27.25. Figure 2 shows the ATLAS $c$-band prediscovery and the earliest detection images of SN 2017ein. The ATLAS observations of SN 2017ein lasted until 2017 July 03. The ATLAS data are reduced and calibrated automatically as described in (Tonry et al. 2018). The photometry was done on the difference images by subtracting pre-explosion templates. A model of the point-spread-function (PSF) was created and fitted to the excess flux sources in the images (as described in Tonry et al. 2018). The photometric calibration was achieved with a custom-built catalogue based on the Pan-STARRS reference catalogues (Magnier et al. 2016), and the magnitudes are in the AB system.

All $B V R I$ CCD images were pre-processed using standard $\mathrm{IRAF}^{2}$ routines, which includes corrections for bias, flat field, and removal of cosmic rays. As SN 2017ein exploded near a spiral arm of the host galaxy, the late-time photometry may be influenced by contamination from the galaxy. We thus use the template images taken on 2018 Jun. 16 and 18 (which correspond to $\sim+390$ days from the discovery) to get better photometry for the AZT images taken at late phases when the supernova was relatively faint. We did not apply template subtraction to the TNT images as the SN is bright relative to the galaxy background when these images were taken. The instrumental magnitudes of both the SN and the reference stars were then measured using the standard point spread function (PSF). These magnitudes were converted to those of the standard Johnson system using the Pan-STARRS reference catalogue $^{3}$. The magnitudes of the comparison stars used for flux calibration, as marked in Figure 1, are listed in Table 1. Although the supernova is not visible in images taken after MJD 58107, we also measured the magnitudes at the supernova site. These measurements provide upper limits of the late-time light curves. The

\footnotetext{
2 IRAF is distributed by the National Optical Astronomy Observatories, which are operated by the Association of Universities for Research in Astronomy, Inc., under cooperative agreement with the National Science Foundation (NSF).

3 http://pswww.ifa.hawaii.edu/pswww/
}

final flux-calibrated magnitudes (Vega) of SN 2017ein from TNT and AZT are shown in Table 7, and the photometric results (AB magnitudes) from ATLAS are reported in Table 8.

SN 2017ein was also observed with the Ultraviolet/Optical Telescope (UVOT; Roming et al. 2005) onboard the Neil Gehrels Swift Observatory (Gehrels et al. 2004). The observations were obtained in uvw1, uvm2, uvw2, $u, b$, and $v$ filters, from 2017 May 27 until 2017 July 21. The Swift/UVOT data reduction is based on that of the Swift Optical Ultraviolet Supernova Archive (SOUSA; Brown et al. 2014). A $3^{\prime \prime}$ aperture is used to measure the source counts with an aperture correction based on an average PSF. Magnitudes are computed using the zeropoints from Breeveld et al. (2011) for the UV and Poole et al. (2008) for the optical and the 2015 redetermination of the temporal sensitivity loss. The photometry is performed on the images subtracted by the template that was obtained on 2018 Jun. 02. The supernova was visible only in uvw1 band but not in uvw2 and uvm2 bands, likely due to the significant reddening that it suffered from the host galaxy (see discussion in Section 4). The Swift-UVOT photometry is listed in Table 9 . The UVOT $b$ - and $v$-band photometry are comparable to those of the ground-based observations around peak in $B$ and $V$ bands, respectively, while the UVOT photometry suffered large uncertainties at later phases.

\subsection{Optical spectroscopy}

Our first spectrum of SN 2017ein was obtained on UT 2017 May 26.57 with the Xinglong 2.16-m telescope of NAOC (hereafter XLT), which is at $\sim 2.5$ days after explosion, assuming that the explosion date is 2017 May 24.0 according to the analysis conducted in Section 6. A sequence of 9 low-resolution spectra were collected with different telescopes including the XLT, the Lijiang 2.4-m telescope (hereafter LJT), the ARC 3.5-m telescope at Apache Point Observatory (hereafter APO 3.5-m), the 2-m Faulkes Telescope North (FTN) of the Las Cumbres Observatory network, and the 9.2-m Hobby-Eberly Telescope (HET). The details of the spectroscopic observations are listed in Table 2.

All spectra were reduced using the standard IRAF routines, which involves corrections for bias, flat field, and removal of cosmic rays. The $\mathrm{Fe} / \mathrm{Ar}$ and $\mathrm{Fe} / \mathrm{Ne}$ arc lamp spectra obtained during the observation night are used to calibrate the wavelength of the spectra, and standard stars observed on the same night at similar airmasses as the supernova were used to calibrate the flux of spectra. The spectra were further corrected for continuum atmospheric extinction during flux calibra- 
Table 1. Magnitudes of Comparison Stars in Field of SN 2017ein.

\begin{tabular}{ccccccc}
\hline \hline Star No. & R.A. & Decl. & $B$ & $V$ & $R$ & $I$ \\
\hline 1 & $11 \mathrm{~h} 52 \mathrm{~m} 40 \mathrm{~s} .50$ & $44^{\circ} 05^{\prime} 49^{\prime \prime} .41$ & $15.848 \pm 0.037$ & $15.067 \pm 0.013$ & $14.611 \pm 0.015$ & $14.194 \pm 0.016$ \\
2 & $11 \mathrm{~h} 52 \mathrm{~m} 44 \mathrm{~s} .22$ & $44^{\circ} 02^{\prime} 20^{\prime \prime} .76$ & $16.490 \pm 0.035$ & $15.774 \pm 0.012$ & $15.354 \pm 0.015$ & $14.927 \pm 0.016$ \\
3 & $11 \mathrm{~h} 52 \mathrm{~m} 48 \mathrm{~s} .69$ & $44^{\circ} 11^{\prime} 01^{\prime \prime} .51$ & $16.879 \pm 0.037$ & $16.442 \pm 0.013$ & $16.173 \pm 0.015$ & $15.865 \pm 0.016$ \\
4 & $11 \mathrm{~h} 52 \mathrm{~m} 56 \mathrm{~s} .69$ & $44^{\circ} 10^{\prime} 50^{\prime \prime} .83$ & $15.874 \pm 0.036$ & $15.166 \pm 0.013$ & $14.750 \pm 0.015$ & $14.337 \pm 0.016$ \\
5 & $11 \mathrm{~h} 53 \mathrm{~m} 07 \mathrm{~s} .08$ & $44^{\circ} 04^{\prime} 30^{\prime \prime} .23$ & $14.386 \pm 0.036$ & $13.843 \pm 0.013$ & $13.516 \pm 0.015$ & $13.149 \pm 0.016$ \\
6 & $11 \mathrm{~h} 53 \mathrm{~m} 16 \mathrm{~s} .23$ & $44^{\circ} 10^{\prime} 13^{\prime \prime} .08$ & $16.880 \pm 0.039$ & $16.009 \pm 0.016$ & $15.504 \pm 0.017$ & $15.052 \pm 0.017$ \\
\hline
\end{tabular}
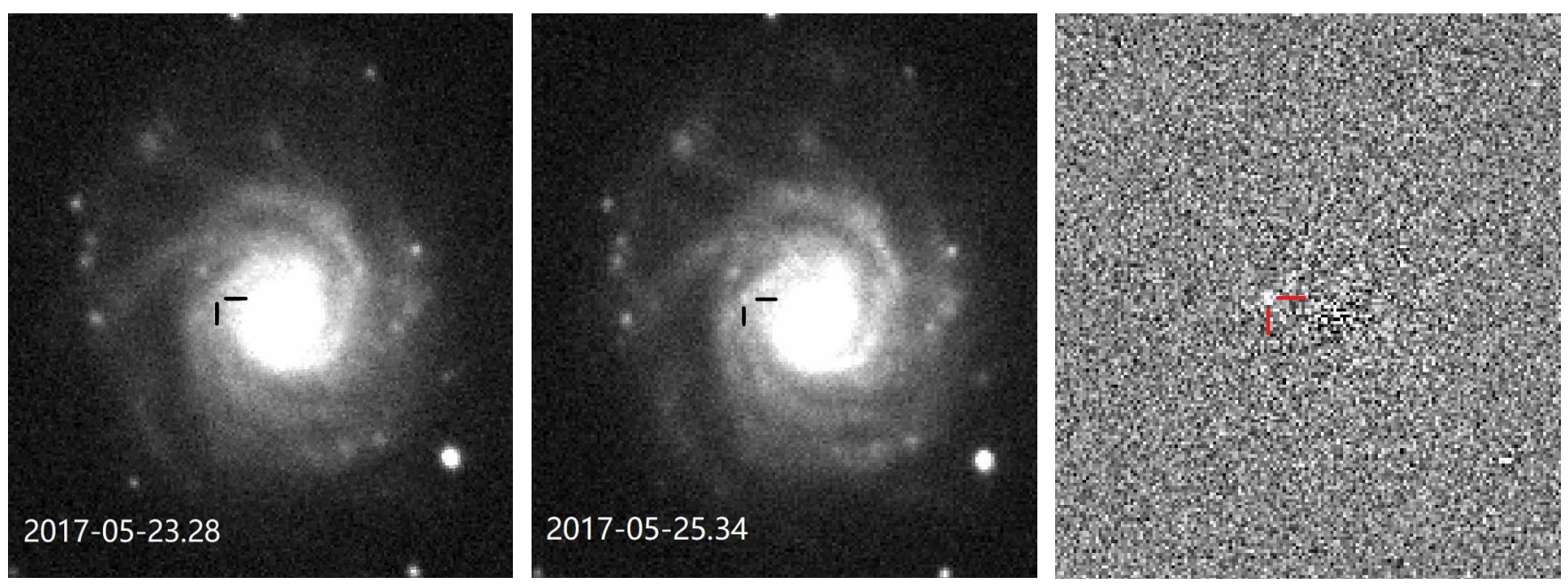

Figure 2. The ATLAS images centering at the galaxy NGC 3938, north is up and west is right. left: The latest non-detection image taken on 2017 May 23.28 in $o$-band. The AB magnitude at the SN position is fainter than 20 mag (after subtracting template); middle: the first detection image of SN 2017ein on 2017 May 25.34 in c-band; right: residual image obtained by subtracting the template from the first-detection image, with the position of the SN marked.

tion, using mean extinction curves obtained at Xinglong Observatory, Lijiang Observatory, Apache Point Observatory, and Haleakala Observatory in Hawaii; moreover, telluric lines were removed from the data.

\section{PHOTOMETRIC PROPERTIES OF SN 2017EIN}

\subsection{Optical and ultraviolet light curves}

Figure 3 shows the AZT and TNT BVRI light curves of SN 2017ein, in comparison with those of SN 2007gr (Chen et al. 2014). The Swift UVOT uvw1- and $u b v$-band light curves are also overplotted. Applying polynomial fits to the data around maximum light, we found that SN 2017ein reached B-band peak of $\mathrm{m}_{B}($ peak $)=15.91 \pm 0.04 \mathrm{mag}$ on MJD 57909.86, and $V$ band peak of $\mathrm{m}_{V}$ (peak $)=15.20 \pm 0.02$ mag at $\sim 3.6$ days later. This gives an estimate of $B_{\max }-V_{\max }$ color as $0.71 \pm 0.05$ mag for SN 2017ein, which is much redder than the typical value for normal SNe Ic. Given the small reddening of $\mathrm{E}(B-V)_{\mathrm{MW}}=0.019 \mathrm{mag}$ due to the Milky Way (Schlafly \& Finkbeiner 2011) and a typical
$B-V$ color of $0.4 \mathrm{mag}$ for normal SNe Ic near the maximum light (Drout et al. 2011), the red color observed for SN 2017ein indicates that it suffered significant reddening due to the host galaxy (see also discussion in Section 4).

In Figure 4, we compare the $B V R I$-band light curves of SN 2017ein to those of other well observed $\mathrm{SNe} \mathrm{Ib} / \mathrm{c}$. SN 2017ein shows light curves that are very similar to SN 2007gr in all four BVRI bands before $\mathrm{t} \sim+60$ days from the peaks. Adopting the explosion date as MJD 57897.0 obtained in Section 6, we find a rise time of $\sim 13.2$ days in the $B$ band and $\sim 16.6$ days in the $V$ band for SN 2017ein. This rise time is longer than that of SN 1994I and SN 2004aw, but similar to that of SN 2007gr and SN 2013ge. Moreover, we also measured the post-peak magnitude decline-rate parameter $\Delta m_{15}$, which is derived as $1.33 \pm 0.07 \mathrm{mag}$ in $B$ and $0.93 \pm 0.04$ mag in $V$ respectively for $\mathrm{SN} 2017$ ein. These values are nearly equal to those measured for SN $2007 \mathrm{gr}$ $\left(\Delta m_{15}(B)=1.31 \mathrm{mag} ;\right.$ Chen et al. 2014) and slightly 
Table 2. Journal of Optical Spectroscopy of SN 2017ein.

\begin{tabular}{ccccccc}
\hline \hline UT & MJD & $t_{e}{ }^{\mathrm{a}}$ & $t_{V}{ }^{\mathrm{b}}$ & Telescope & Instrument & Exposure time (s) \\
\hline $2017 / 05 / 26.57$ & 57899.57 & 2.6 & -13.9 & BAO 2.16 m & BFOSC+G4 & 3600 \\
$2017 / 05 / 30.63$ & 57903.63 & 6.6 & -9.9 & BAO 2.16 m & BFOSC+G4 & 3000 \\
$2017 / 05 / 31.60$ & 57904.60 & 7.6 & -8.9 & BAO 2.16 m & BFOSC + G4 & 3000 \\
$2017 / 06 / 15.15$ & 57919.15 & 21.7 & +5.2 & HET 9.2 m & LRS2 & 1500 \\
$2017 / 06 / 20.56$ & 57924.56 & 27.5 & +11.0 & BAO 2.16 m & BFOSC+G4 & 2700 \\
$2017 / 06 / 23.29$ & 57927.29 & 30.3 & +13.8 & FTN 2.0 m & FLOYDS & 2700 \\
$2017 / 06 / 28.28$ & 57932.28 & 35.3 & +18.8 & FTN 2.0 m & FLOYDS & 2700 \\
$2017 / 07 / 29.55$ & 57963.55 & 66.5 & +50.1 & LJO 2.4 m & YFOSC+G10 & 2100 \\
$2017 / 08 / 21.04$ & 57987.04 & 90.0 & +73.6 & APO 3.5 m & DIS+B300\&R400 & 1800 \\
\hline
\end{tabular}

${ }^{a}$ Days after explosion.

${ }^{b}$ Phase relative to the $V$-band maximum.

larger than those for SN 2013ge $\left(\Delta m_{15}(B)=1.11 \mathrm{mag}\right.$; Drout et al. 2016). The full light-curve parameters in different bands are listed in Table 3. Our observations did not cover the period from t $\sim 60-190$ days after $V$-band maximum due to solar conjunction. The later observations restarted on MJD 58107, corresponding to a phase of $\mathrm{t} \sim+193 \mathrm{~d}$ with respect to the $V$ maximum. The supernova became too faint to be detectable except in $R$ - and $I$-bands, where SN 2017ein is found to be apparently fainter than SN 2007gr at this phase. Due to the absence of observations during $\mathrm{t} \sim+60-190 \mathrm{~d}$, it is unclear when the light curves of SN 2017ein started to deviate from those of SN $2007 \mathrm{gr}$.

Figure 4 shows the comparison of SN 2017ein with some well-observed SNe Ibc including SNe 1994I, 2002ap, 2004aw, 2007gr, 2008D, and 2013ge. The upper limits showing obvious large deviations from the evolutionary trend are due to improper subtraction and bad weather and are not plotted. The overall light curve evolution of SN 2017ein shows close resemblance to that of SN 2007gr at $\mathrm{t}<+60$ days from the peak, while in the nebular phases it clearly shows a much faster decline than SN 2007gr and other SNe Ib/c with similar light curve shapes near maximum light. On day MJD 58107, SN 2017ein is found to be fainter than SN 2007gr by $\sim 0.9 \mathrm{mag}$ in $I$-band and $\sim 0.4 \mathrm{mag}$ in $R$-band, respectively. The relatively slower decline in the $R$ band is probably due to a contribution of $\mathrm{O} \mathrm{I}$, $\mathrm{O}$ II, and Ca II emission in the nebular phases. For SN 2007gr, Chen et al. (2014) found that it dimmed at a pace faster than the ${ }^{56} \mathrm{Co}$ decay in all bands after $t \sim+80 \mathrm{~d}$ from the peak, which can be explained with an increase of escape rate of $\gamma$-rays at this phase; and its $R$-band light curve also showed a relatively slower evolution than other bands. Thus, the faster decay seen for the late-time light curves of SN 2017ein could be due to larger escape rate of the $\gamma$ photons, suggestive of a low ejecta mass for SN 2017ein (see also discussion in Section 6). Alternatively, the newly formed dust in the cooling ejecta may also shed light from the supernova.

The color evolution of SN 2017ein and the comparison sample is presented in Figure 5, where all color curves have been corrected for the total reddening to the supernova. The colors derived from the earliest two spectra are also included in the plot. For SN 2017ein, a total reddening of $\mathrm{E}(B-V) \sim 0.42 \mathrm{mag}$ derived in the next section is removed from the color curves. As can be seen, all color curves seem to follow a similar trend, evolving bluewards from $t \sim 2$ weeks to a few days before the $V$ band maximum. Note that the $B-V$ and $B-R$ colors of SN 2008D are very blue at $\mathrm{t} \lesssim 2$ weeks before $V$-band maximum and they evolve redwards quickly, suggestive of an initial high temperature that can been interpreted as shock heating or interaction (Modjaz et al. 2009; Mazzali et al. 2008). This feature is also observed in the color evolution of SN 2017ein, suggesting that its earlytime emission may have also experienced a cooling stage of shock breakout emission. We will further investigate this feature in Section 6.

After reaching the bluest color, all color curves become progressively red until 3 weeks after maximum, followed by a plateau phase. The $B-V$ color curves of different $\mathrm{SNe} \mathrm{Ib} / \mathrm{c}$ tend to converge at 0.5 mag near maximum light, though they show relatively large scatter before and after the peak. The $V-R$ color shows a similar trend, and it tends to converge at $\sim 0.2-0.3$ mag at about 10 days from $V$ band maximum as suggested by Drout 


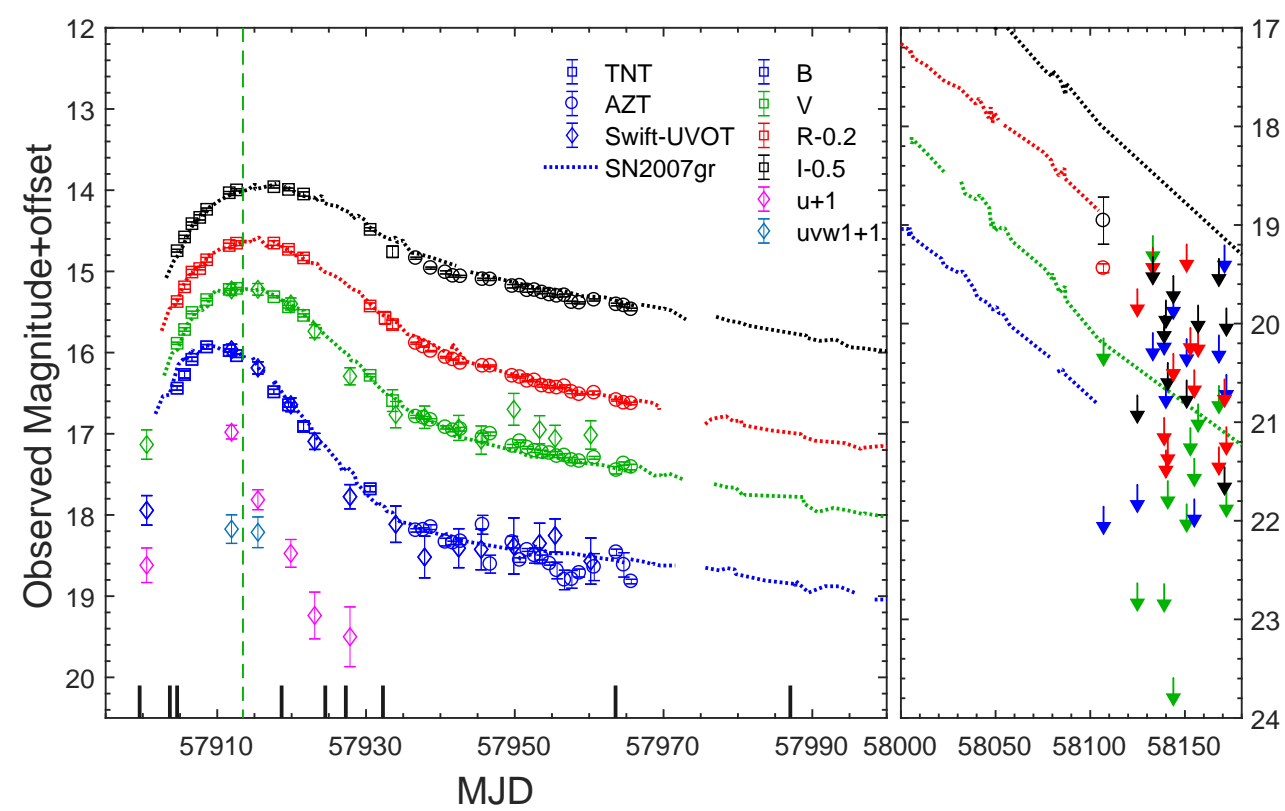

Figure 3. $B V R I$ light curves of SN 2017ein, obtained with the 0.8-m Tsinghua-NAOC telescope, AZT-22 1.5-m telescope, and Swift UVOT. The vertical dashed line marks the maximum time in the $V$ band. The ticks at the bottom mark the epoches with spectroscopic observations. Also plotted are the BVRI light curves of SN 2007gr (Chen et al. 2014). The light curves of SN 2007gr are shifted in time and magnitudes to match the corresponding light curve peaks of SN 2017 ein.

Table 3. Light-curve Parameters Obtained for SN 2017ein.

\begin{tabular}{ccccc}
\hline \hline band & peak date (MJD) & peak obs. mag. & peak abs. mag. & $\Delta m_{15}(\mathrm{mag})$ \\
\hline$B$ & $57909.86 \pm 0.24$ & $15.91 \pm 0.04$ & $-17.20 \pm 0.38$ & $1.33 \pm 0.07$ \\
$V$ & $57913.49 \pm 0.20$ & $15.20 \pm 0.02$ & $-17.47 \pm 0.35$ & $0.93 \pm 0.04$ \\
$R$ & $57914.67 \pm 0.28$ & $14.81 \pm 0.05$ & $-17.54 \pm 0.33$ & $0.75 \pm 0.09$ \\
$I$ & $57916.32 \pm 0.30$ & $14.43 \pm 0.04$ & $-16.93 \pm 0.32$ & $0.61 \pm 0.09$ \\
\hline
\end{tabular}

et al. (2011). SN 2017ein shows color evolution that overall is quite similar to SN $2007 \mathrm{gr}$.

\subsection{The early light curves from ATLAS}

The survey observation of ATLAS is operated in two specifically designed bands, ATLAS-cyan $(c)$ and ATLAS-orange (o) (Tonry et al. 2018). Figure 6 shows the transmission curves of these two filters and the broadband $B V R I$-band filters. One can see that the ATLAS $o$ and $c$ filters have similar effective wavelengths to the broadband $R$ and $V$ filters, respectively. To examine the difference between them, we calculate the synthetic $c-V$ and $o-R$ colors using the spectra of SN 2017ein presented in this paper, and those from Van Dyk et al. (2018). The results are shown in Figure 7. The $V$-band magnitudes appear slightly brighter than those in $c$-band by $\sim 0.2 \mathrm{mag}$, and this difference tends to be smaller in the early and late phases (see the left panel of Figure 7). The ATLAS-o magnitude differs from the $R$-band value at the same phase by $\sim 0.15 \mathrm{mag}$, as shown in the middle panel of Figure 7; and in very early phase this difference is less than $0.1 \mathrm{mag}$. In the right panel of Figure 7, we show the evolution of the ATLAS $c-o$ color for SN 2017ein, with the $c$-band magnitudes being fainter than the $o$-band by $0.2-0.8$ mag. A low-order polynomial fit is applied to the $c-V, o-R$, and $c-o$ color evolutions, and the best-fit result is then used to interpolate the magnitudes in different bands when necessary.

Figure 8 shows the ATLAS o-band light curves (AB magnitude) of SN 2017ein, together with those converted from the AZT and TNT $R$-band photometry using the $o-R$ relationship shown in Figure 7. In the conversion, we added a systematic error of $0.03 \mathrm{mag}$ 


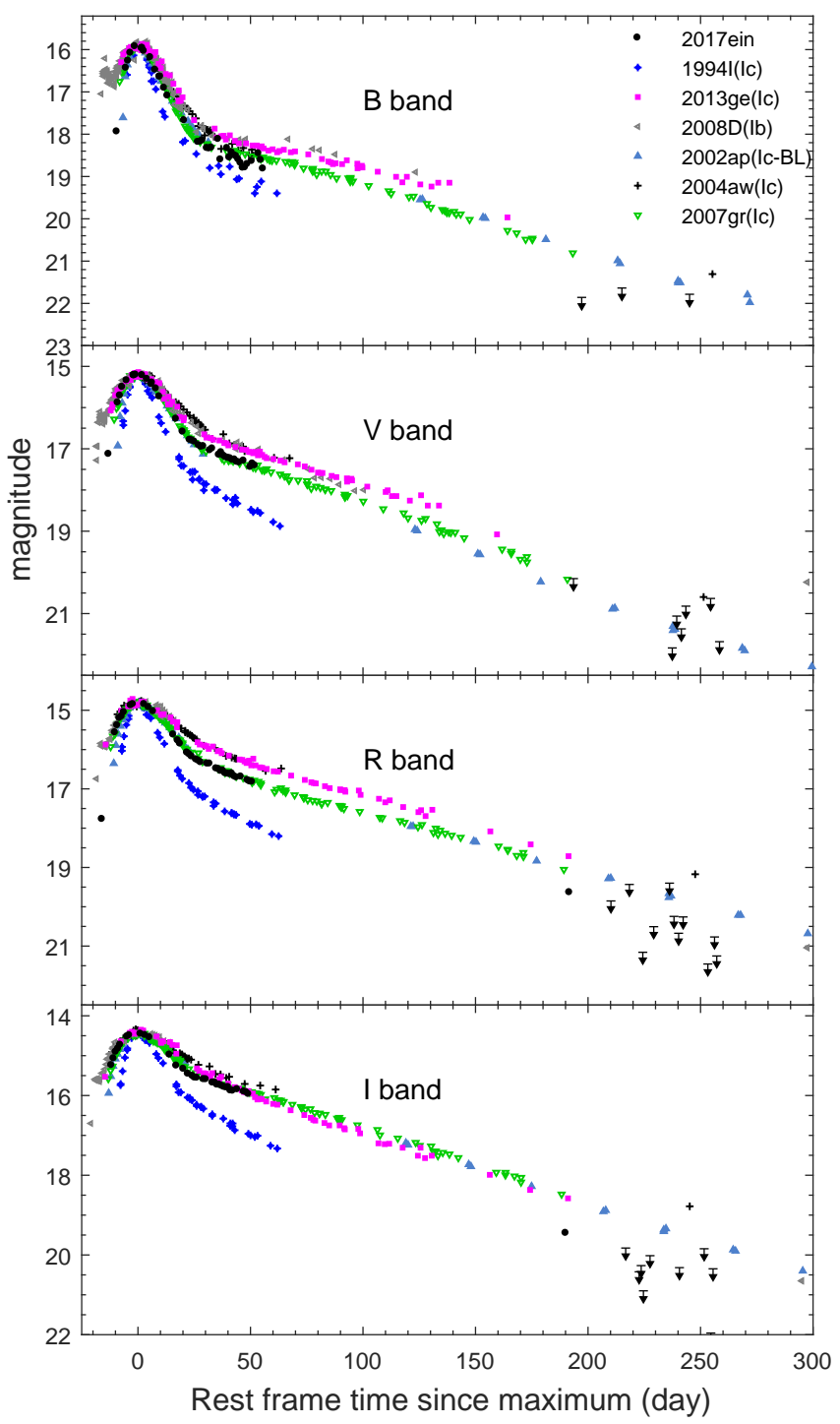

Figure 4. Comparison of $B V R I$ light curves of SN 2017ein with other SNe Ib/c: SN 1994I, SN 2002ap, 2004aw, 2007gr, SN 2008D and SN 2013ge. The light curves are shifted in time and magnitude to match the peaks of SN 2017ein. Data references: SN 1994I (Richmond et al. 1996; Yokoo et al. 1994), SN 2002ap (Foley et al. 2003), SN 2004aw (Taubenberger et al. 2006), SN 2007gr (Chen et al. 2014), SN 2008D (Mazzali et al. 2008; Modjaz et al. 2009; Bianco et al. 2014; Brown et al. 2014), and SN 2013ge (Drout et al. 2016).

to the converted magnitude errors. And the converted light curve is shifted by 0.13 mag to match the peak of the $o$-band light curve. The earliest prediscovery detection from the ATLAS $c$-band observation, taken on 2017 May 25, is also plotted for comparison. In this plot, we also include the magnitude derived from our first spectrum taken on 2017 May 26.57. This spectrum was flux-calibrated using the first $R$-band photometry from AZT (taken on 2017 May 25.77) and the earliest
ATLAS o-band photometry (taken on 2017 May 27.26). The earliest $R$-band photometry was reported in Im et al. (2017) with a magnitude of $17.7 \mathrm{mag}$ on MJD 57898.77. We obtained the raw images through private communication and reduced the data with our pipeline ZrutyPhot (Mo et al. in prep.) and obtain a magnitude of $17.78 \pm 0.01 \mathrm{mag}$. This measurement is converted to the $o$-band magnitude and included in our analysis as well. The latest non-detection is from the 1-m telescope at Deok-Heong Optical Astronomy Observatory (DOAO) in South Korea, which gives an $R$-band limit of $>18.4$ mag from images taken on 2017 May 24.63 (Im et al. 2017), which is transformed to the AB system and also plotted in Figure 8.

These early-time data can be used to constrain the time of first-light for SN 2017ein. Using the data points before MJD 57907 and adopting a function of luminosity evolution as $L(\lambda) \propto t^{n}$ in the fit, we find the explosion date of MJD $57897.2 \pm 0.6$ by fitting the early the TNT and AZT $R$-band data. To get better data sample, especially at early times, we also combine the converted $R$-band data with the ATLAS $o$-band data together to infer the explosion time as MJD $57896.8 \pm 0.3$ (see Figure 8). However, we caution that such a combined analysis may suffer some uncertainty due to that the conversion between these bands is not very accurate. These estimated dates are very close to the nondetection date MJD 57896.77 presented by $\mathrm{Im}$ et al. (2017), with $R>19.9$ mag. Thus, the explosion date should be near $\mathrm{MJD}=57896.9$, with a $1-\sigma$ error of 0.3 day. The corresponding power-law index for the above fits is $n=1.36$ and 1.51 , respectively, which are smaller than the typical value of $n=2$ for the fireball model. A more detailed analysis of the explosion date is presented in Section 6, which makes use of the whole range of the light curves.

As SN 2017ein tends to have relatively bluer $c-o$ color in the early phase after explosion (see Figure 7), we assume a $c-o$ color of $\sim 0.3 \mathrm{mag}$ according to the $c-o$ evolution (Figure 7 ). The $o$-band magnitudes are thus inferred from the corresponding $c$-band photometry and are shown as empty circles in Figure 8. When the supernova was first detected in $c$ band at $t \sim-15.15$ days from the maximum light, it showed an excess emission beyond the power-law model. This serves as an indication for possible detection of shock breakout cooling as addressed in section 6 .

\section{DISTANCE, REDDENING AND ABSOLUTE MAGNITUDE}

The distance to the host galaxy of NGC 3938 (and hence SN 2017ein) was estimated by three methods. 

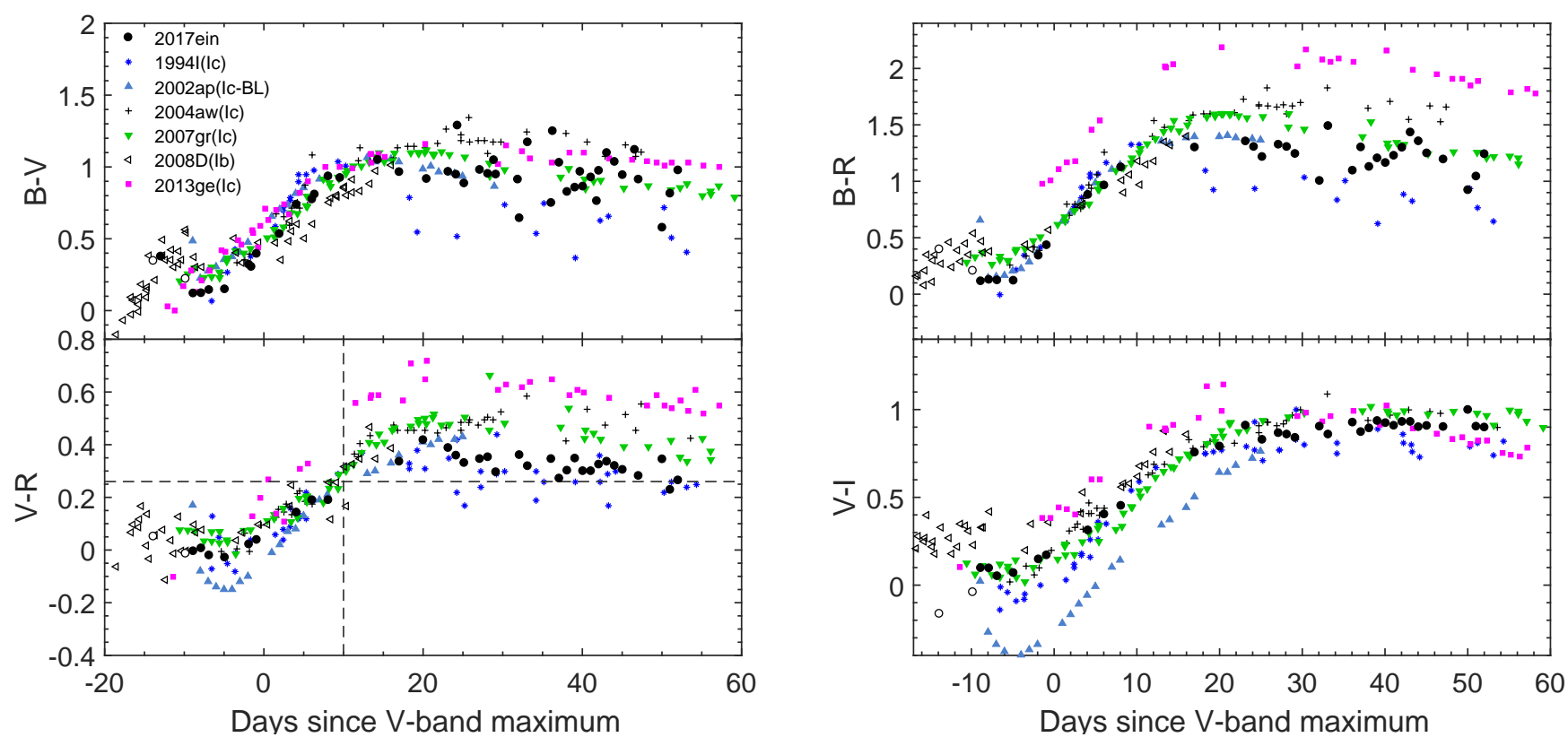

Figure 5. Color evolution of SN 2017ein and some well-observed SNe Ic, all corrected for the Galactic and host-galaxy reddening. The color curve of SN 2008D (SN Ib) is overplotted for comparison. The open circles represent the synthetic color derived from the spectra. The dashed lines mark that the $V-R$ color tends to have a uniform value of $\sim 0.26$ in Drout et al. (2011) at $\mathrm{t} \sim+10$ days from the $V$-band maximum. Data references are the same as in Figure 5.

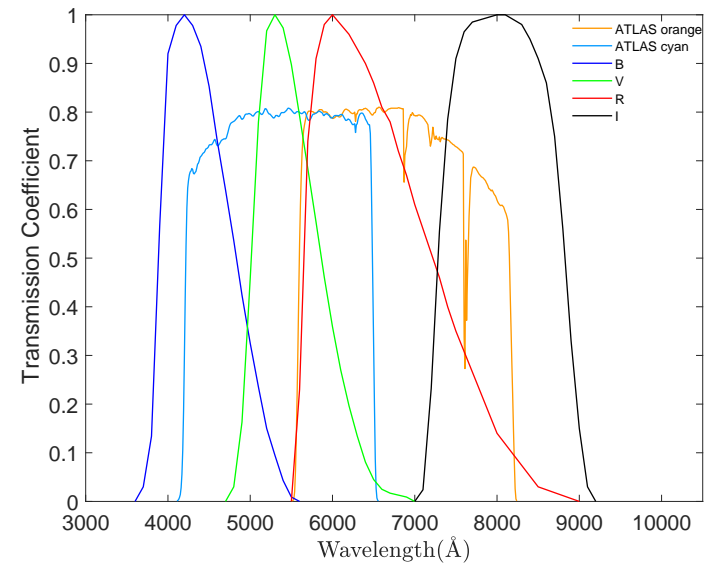

Figure 6. The transmission functions of the two ATLAS filters, compared with the Johnson $B V R I$ filters.

One is based on the standardization of SNe II through an empirical relation between photospheric magnitude and color (Rodríguez et al. 2014), which is calibrated using SNe II with Cepheid distances. With the observed parameters of the SN IIP exploded in NGC 3938, SN 2005ay, a distance of $31.75 \pm 0.24 \mathrm{mag}$ and $31.70 \pm 0.23 \mathrm{mag}$ can be derived for this galaxy, by using the $V$ and $I$-band data respectively. While a smaller value of $31.27 \pm 0.13 \mathrm{mag}$ was obtained when using correlation between luminosity and photospheric velocity of SNe IIP (Hamuy \& Pinto 2002; Poznanski et al. 2009). A smaller value of $31.15 \pm 0.40 \mathrm{mag}$ can be inferred from the Tully-Fisher relation if the Hubble constant is adopted as $75 \mathrm{~km} \mathrm{~s}^{-1} \mathrm{Mpc}^{-1}$ (Tully 1988). In contrast to adopting a distance modulus in a range from 31.15 to 31.75 mag (see Van Dyk et al. 2018), we adopt an average (not weighted) value of $31.38 \pm 0.30$ mag for the distance modulus throughout this paper.

The red peak $B-V$ color and the red spectra at early epoches both suggest that SN 2017ein suffered severe reddening from the host galaxy. We estimated the exact reddening through the $\mathrm{Na}$ I $\mathrm{D}$ absorption detected in the spectra. A moderately strong NaID absorption with an equivalent width (EW) of $2.48 \pm 0.35 \AA$ can be inferred from the spectra of SN 2017ein taken by 2-m FTN telescope with good signal-to-noise ratio (SNR). Based on the relation of $\mathrm{E}(B-V)$ and $\mathrm{EW}$ of $\mathrm{Na}$ I $\mathrm{D}$ (Turatto et al. 2003), i.e., $\mathrm{E}(B-V)=0.16 \times \mathrm{EW}(\mathrm{Na}$ I D), the host-galaxy reddening is thus estimated to be $\mathrm{E}(B-V)_{\text {host }}=0.40 \pm 0.06$ mag.

On the other hand, a systematic statistical study of $\mathrm{SNe} \mathrm{Ib/c} \mathrm{light} \mathrm{curves} \mathrm{(Drout} \mathrm{et} \mathrm{al.} \mathrm{2011)} \mathrm{concludes} \mathrm{that}$ the $V-R$ color of $\mathrm{SNe} \mathrm{Ib} / \mathrm{c}$ clusters at $\sim 0.26 \pm 0.06 \mathrm{mag}$, as shown by the dashed lines in the $V-R$ color plot of Figure 5. To match this trend, the observed $V-R$ color of SN 2017ein has to be shifted by an amount of about $0.4 \mathrm{mag}$, which is consistent with that derived 

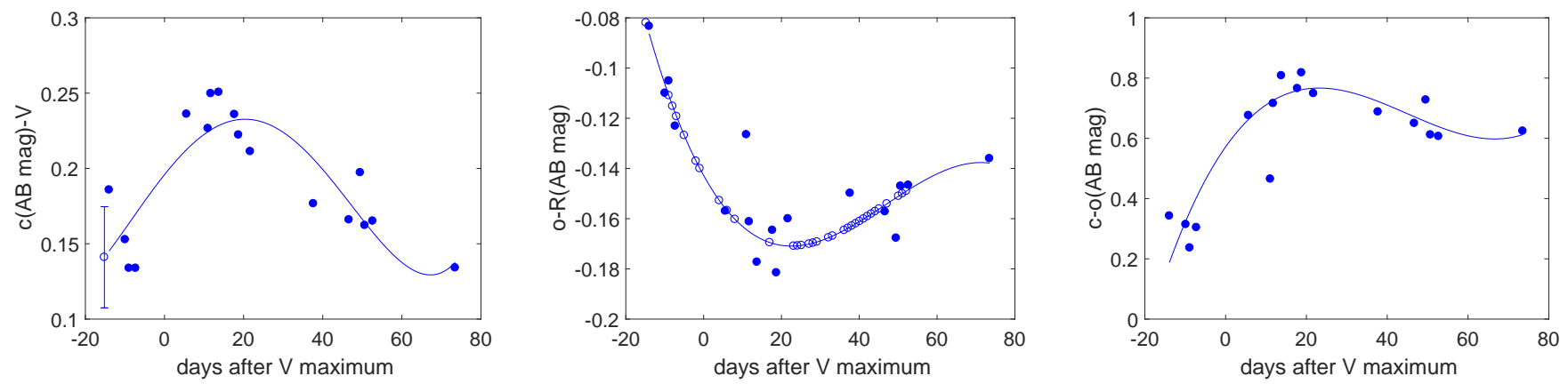

Figure 7. left: The $c-V$ (left), $o-R$ (middle), and $c-o$ (right) color evolutions of SN 2017ein, obtained using the supernova spectra presented in this paper and those taken from Van Dyk et al. (2018). The solid lines in each panel are the lower-order polynomial fits to the observed data. The open circle with an error bar is the $c-V$ color at the ATLAS- $c$ photometry epoch which is used to transform the $c$ magnitude to $V$ magnitude. The open circles in the middle panels represent the fitting values at the $R$ band photometry epochs, which are used to convert the $R$ band magnitudes to $o$ magnitudes.

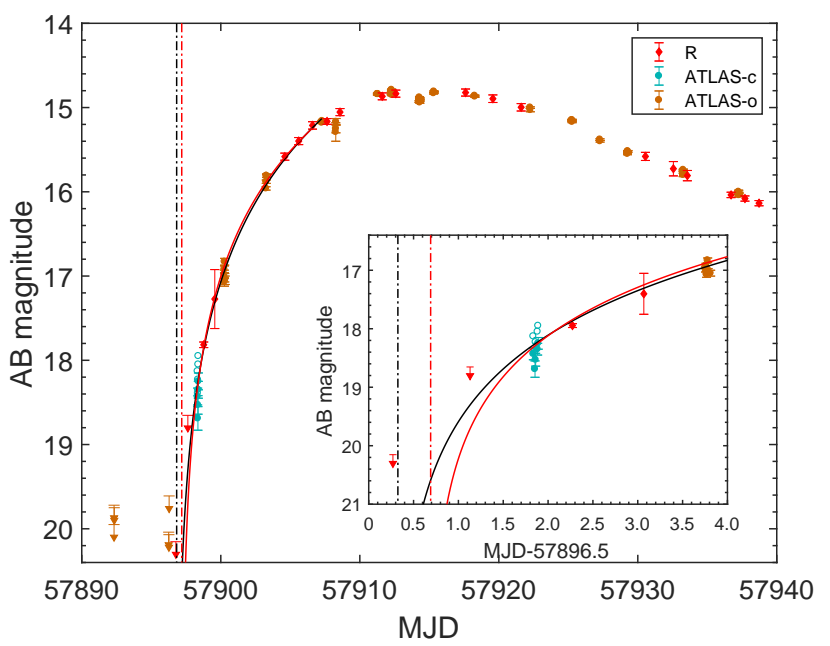

Figure 8. The ATLAS $c$ and $o$-band light curves of SN 2017ein. The $R$-band light curves from the TNT and AZT transferred to $o$-band light curves using the relation derived in Figure 7 are also overplotted. The detection limits from ATLAS and the report in Im et al. (2017) are shown by red downward arrows. The red line shows the power-law fit $\left(f \propto t^{n}\right)$ to the pre-maximum $R$-band data, while the black line represents the fit to the combined data from ATLAS, AZT, and TNT. The corresponding best-fit explosion dates are shown by the vertical dot-dashed lines. The empty symbols in blue show the estimated magnitudes in $o$ band at epochs with $c$-band observations. The embedded plot shows the zoomed-in early-time light curve and the fitting.

from the Na I D absorption. Adopting the extinction coefficient $\mathrm{R}_{V}=3.1$, the total extinction is estimated to be $\mathrm{A}_{V}=1.29 \pm 0.18 \mathrm{mag}$ for SN 2017ein. With the distance and extinction values derived above, we estimate the absolute $V$-band peak magnitude of SN 2017ein as $-17.47 \pm 0.35 \mathrm{mag}$. This value is within the range of normal SNe Ib/c, similar to SN 2007gr, SN 2013ge, SN 1994I etc., but fainter than SN 1998bw, SN 2004aw and LSQ14efd. In the comparison sample, SN 1998bw is the brightest, while SN 2008D is the faintest.

\section{OPTICAL SPECTRAL PROPERTIES}

\subsection{Spectral classification}

The complete spectral evolution of SN 2017ein is shown in Figure 9, spanning the phase from $t \sim-13.9$ days to $t \sim+73.6$ days relative to the $V$-band maximum light. The earliest spectrum of SN 2017ein is characterized by a prominent broad absorption line near $6200 \AA$ which showed rapid evolution and split into two absorption features at $6100 \AA$ and $6250 \AA$ a few days later. This feature in earliest spectrum can be due to a combination of SiII $\lambda 6355$ and CII $\lambda 6580$ absorptions. Prominent broad absorption features can also be seen near $7400 \AA$ and $8100 \AA$ which can be attributed to the OI triplet and the CaII NIR triplet, respectively. The weak absorption near $6950 \AA$ could also be due to CII $\lambda 7235$ instead of HeI $\lambda 7267$, as it disappeared near maximum light. For a $\mathrm{SN} \mathrm{Ib}$, the intensity of the main helium features will increase with time. Thus, the identification of the above features give us confidence to classify SN 2017ein as a type Ic supernova. This classification is further justified by the fact that it closely resembles two typical SNe Ic, SN 2007gr and SN 2013ge, as presented in Section 5.2.

\subsection{Spectral evolution}

In this subsection, we examine the spectral evolution of SN 2017ein in comparison with other $\mathrm{SNe} \mathrm{Ib} / \mathrm{c}$ at several phases. Most of the spectra of the comparison $\mathrm{SNe}$ are obtained via the Open Supernova Catalog ${ }^{4}$ (OSC; Guillochon et al. 2017). All spectra have been corrected for host-galaxy redshift and the total line-of-sight ex-

\footnotetext{
${ }^{4}$ https://sne.space
} 


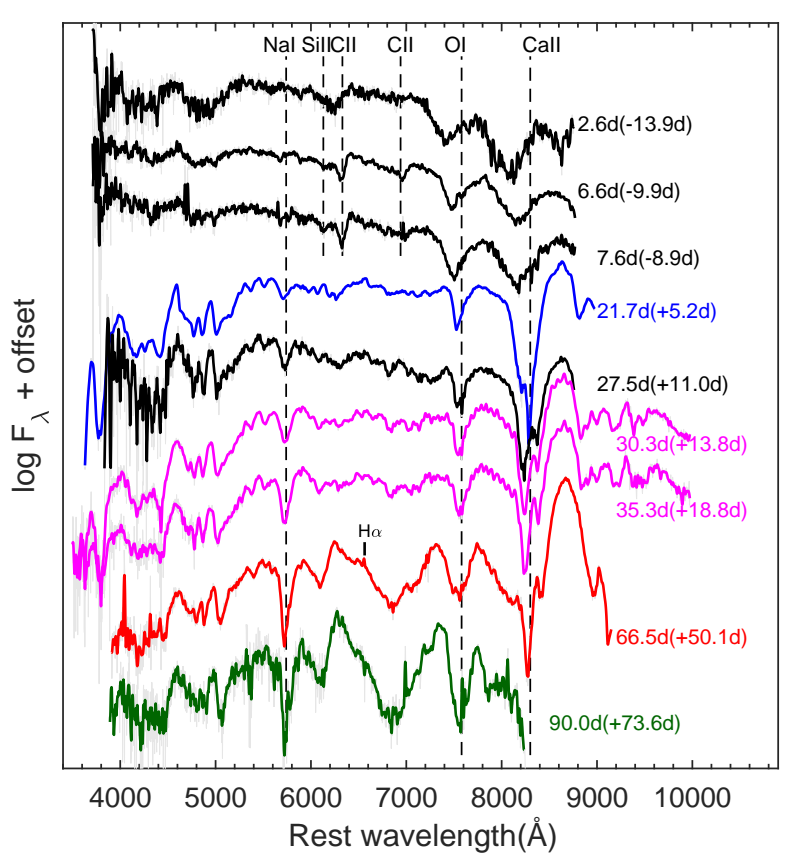

Figure 9. Optical spectra of SN 2017ein. Different colors indicate spectra taken by different telescopes: XLT (black), HET (blue), FTN (magenta), LJT (red), and APO 3.5-m telescope (dark green). The spectral wavelength is in the rest-frame of the host galaxy (corrected for a redshift $\mathrm{z}=0.0027$ ), and the flux is plotted in logarithmic scale and shifted vertically for better display. The numbers beside each spectrum shows the day after explosion and numbers in bracket represent the phase in days relative to the $V$-band maximum. The positions of some noticeable absorption features are marked by dashed lines. The $\mathrm{H} \alpha$ line from the host galaxy is marked out.

tinction using the extinction law of the Milky Way with $\mathrm{R}_{V}=3.1$ (Gordon et al. 2003).

Figure 10 shows the comparison of pre-maximum spectra. The broad absorption feature near $6200 \AA$ at $\mathrm{t} \sim$ $-13.9 \mathrm{~d}$ evolved into two separate narrow absorption features at $\mathrm{t} \sim-10 \mathrm{~d}$. Assuming that these two features correspond to SiII $\lambda 6355$ and CII $\lambda 6580$ absorptions, they have an expansion velocity of about $10,500 \mathrm{~km} \mathrm{~s}^{-1}$ and $12,000 \mathrm{~km} \mathrm{~s}^{-1}$, respectively. The identification of prominent CII $\lambda 6580$ absorption in the $t \sim-10$ day spectrum is supported by the presence of another absorption feature near $6950 \AA$ that can be attributed to CII $\lambda 7235$ with a similar blueshifted velocity. This is also confirmed by the SYNAPPS fit ${ }^{5}$, with input of CII, OI,

\footnotetext{
5 SYNAPPS (Thomas et al. 2011) is a spectrum fitting code based on the supernova synthetic-spectrum code $\mathrm{SYN}++$, which is rewrite of the original SYNOW code in modern $\mathrm{C}++$.
}

NaI, MgII, SiII, CaII, and the singly-ionized iron group elements. The fitting result is shown in Figure 11. Thus in the $\mathrm{t} \sim-13.9$ day spectrum, the SiII and CII lines likely blended together. Applying a double gaussian fit to decompose these two features, they are found to have velocities of $\sim 14,000 \mathrm{~km} \mathrm{~s}^{-1}$ (SiII $\left.\lambda 6355\right)$ and $\sim 16,000$ $\mathrm{km} \mathrm{s}^{-1}$ (CII $\lambda 6580$ ), respectively.

The pre-maximum spectra features of SN 2017ein closely resembles those of SN 2007gr and SN 2013ge, which are all characterized by prominent, narrow CII $\lambda 6580$ absorption and weak SiII $\lambda 6355$ absorption compared with SN 1994I. The presence of high-velocity carbon in SN 2017ein indicates that the outer part of its progenitor star should have abundant carbon, consistent with a WC-type Wolf-Rayet progenitor star that has lost its $\mathrm{H}$ and $\mathrm{He}$ envelopes and remains a bare $\mathrm{CO}$ core before explosion.

The comparison of the post-maximum spectra is shown in Figure 12. A late-time spectrum of type IIb SN 1993J is also overplotted. As one can see, SN 1993J has a strong $\mathrm{H} \alpha$ line near $6150 \AA$, and the type Ib SN 2004gq and SN 2008D have several significant lines at $4300 \AA, 5600 \AA, 6500 \AA$ and $6800 \AA$ which can be attributed to HeI. All these features are absent in SN 2017ein. After maximum light, the spectra of SN 2017ein are also similar to those of SN 2007gr and SN 2013ge except for the CaII NIR triplet. As can be seen in Figure 12, the CaII absorption feature in SN 2017ein splits into two narrow lines since t $+19 d$, which is not seen in other comparison $\mathrm{SNe} \mathrm{Ib/c.} \mathrm{This} \mathrm{is}$ also seen in the late spectra of SN 1993J (Barbon et al. 1995). In previous studies, these two lines are both attributed to the CaII NIR triplet. In most cases, the SN photosphere is optically thick to absorption lines, and thus the adjacent lines are likely to blend and become unresolved. One example is the CaII $\lambda \lambda \lambda 8498$, 8542,8662 , which usually blend together into one broad line. These lines get narrower when the photosphere becomes more transparent and recedes to lower velocities. In this case, the line CaII $\lambda 8662$ splits from the other two CaII NIR lines. Another understanding of the split feature is that the absorption line on the right is not related to CaII NIR triplet but be due to CI $\lambda 8727$ as the neutral carbon line may become stronger as a result of photospheric cooling.

\subsection{Velocity evolution}

We measured the ejecta velocity inferred from different absorption lines in the spectra of SN 2017ein. Figure 13 shows the velocity evolution of FeII $\lambda 5169$, SiII $\lambda 6355$, CII $\lambda 6580$, OI $\lambda 7774$, and CaII NIR triplet lines. The velocity of CaII NIR triplet is obviously higher than other 


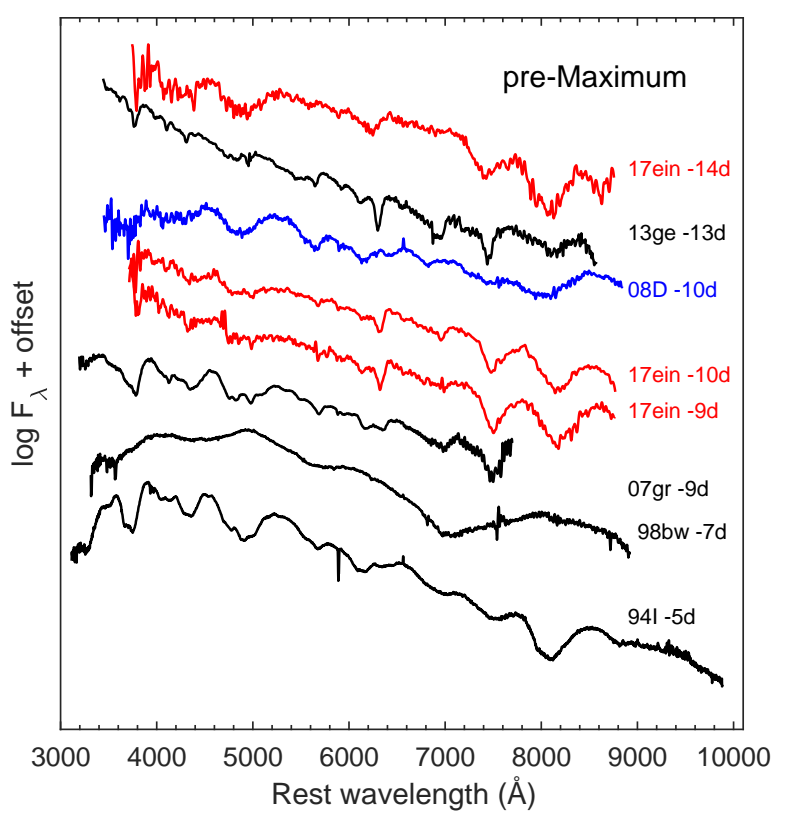

Figure 10. The early-phase spectra of SN 2017ein, compared with those of SN 1994I (Filippenko et al. 1995), SN 1998bw (Patat et al. 2001), SN 2007gr (Valenti et al. 2008), SN 2008D (Modjaz et al. 2009), and SN 2013ge (Drout et al. 2016). Spectra of SN 2017ein are plotted with red color. Different colors represent different SN types: black for Ic, blue for Ib, and magenta for IIb.

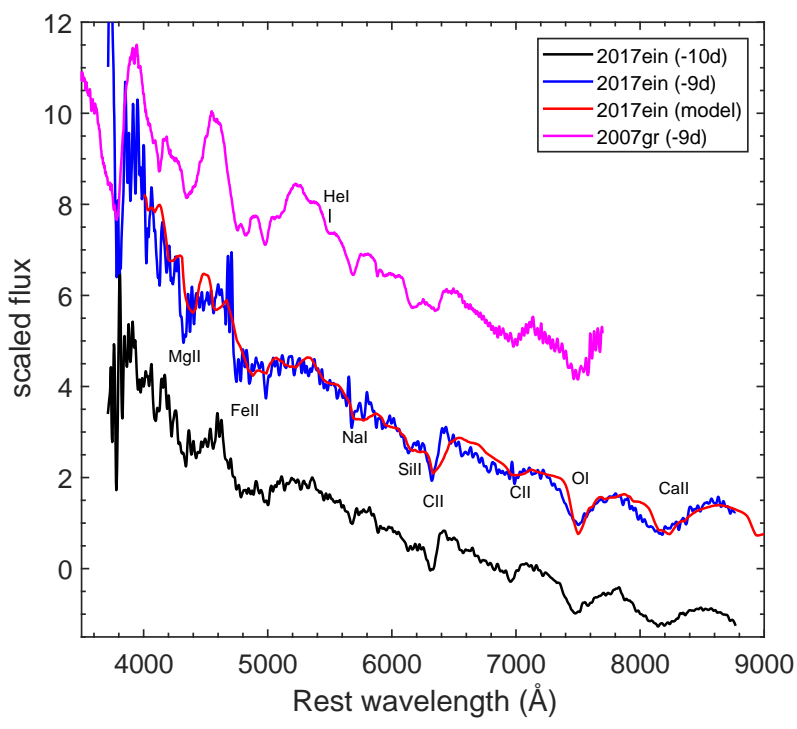

Figure 11. SYNAPPS fit to the spectrum of SN 2017ein at $\mathrm{t}=-9 \mathrm{~d}$ relative to $V$-band maximum. Also plotted the spectrum at $-10 \mathrm{~d}$ and spectrum of SN 2007gr at $\mathrm{t}=-9 \mathrm{~d}$ relative to $V$-band peak (Valenti et al. 2008). The HeI feature in the spectrum of SN 2007gr proposed by Chen et al. (2014) is marked out.

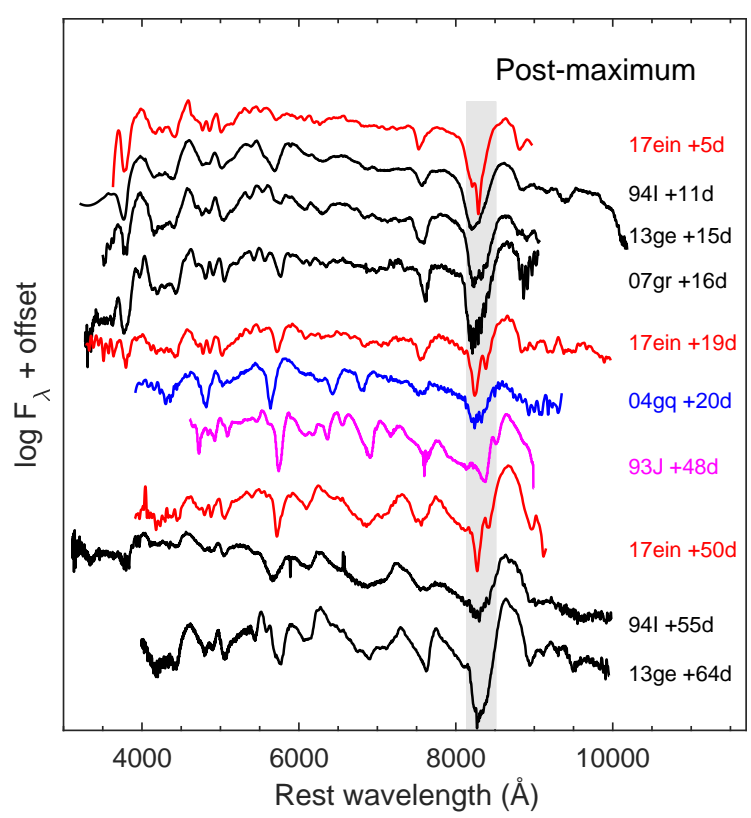

Figure 12. Comparison of the post-peak spectra between SN 2017ein and other SNe Ib/c. The CaII NIR triplet feature is marked by grey shaded area. The line colors are the same as in Figure 10. Data references: SN 1993J (Barbon et al. 1995), SN 1994I (Filippenko et al. 1995), SN 2004gq (Modjaz et al. 2014), SN 2007gr (Valenti et al. 2008), and SN 2013ge (Drout et al. 2016).

lines, which is common for $\mathrm{SNe}$ since calcium has a larger opacity at the same radius and CaII absorption lines thus traces the outer layers of the ejecta at higher velocity.

Figure 14 compares line velocities with some representative SNe Ic, including SNe 1994I, 1998bw, 2002ap, 2004aw, 2007gr, and 2013ge. It is apparent that SNe Ic-BL have much higher SirI velocity from very early phases until maximum light. The ejecta velocity of SN 2017ein shows similar evolution to other normal SNe Ic like SN 2007gr and SN 2013ge, which drops rapidly in about 10 days from explosion and then remains roughly constant in later phases. Among SN 2013ge, SN 2007gr and SN 2017ein, SN 2007gr has the lowest SiII and NaI velocity in the early fast-decreasing stage. But the NaI velocity of SN 2007gr shows a reincreasing trend at later epochs. In comparison with other SNe Ic of our sample, SN 2017ein has a lower CaII velocity, e.g., $\sim 12,000 \mathrm{~km} \mathrm{~s}^{-1}$ versus $\sim 14,000 \mathrm{~km}$ $\mathrm{s}^{-1}$ at around maximum light. This difference may be caused in part by a different method of measuring the line velocity. In SN 2017ein, the CaII absorption splits into two narrower lines, and we measured the velocity from CaII $\lambda 8538$ absorption instead of treating it as a blended trough of $\lambda 8571$. Whereas we can not perform 
such a measurement for other SNe Ic of our sample because their CaII NIR features blended into one broad absorption feature.

It is noteworthy that the velocity of the $\mathrm{Na}$ I D line of SN 1994I showed an obvious increase from $t \sim 27$ days to $t \sim 40$ days from the explosion and reached a velocity plateau after that. The NaI velocity inferred for SN 2007gr seems to have a similar trend of evolution, but with much lower plateau velocity. As discussed in Hunter et al. (2009), this is probably related to the combination of NaII to NaI or the departure from LTE to NLTE, although this trend could be also caused by contamination with HeI $\lambda 5876$. The latter scenario gets support from recent studies that residual He may exist in some SNe Ic (e.g. Chen et al. 2014; Prentice et al. 2018). While Chen et al. (2014) suggested that in the early-time spectra of SN $2007 \mathrm{gr}$, the feature near $5500 \AA$, bluewards of Na I D line, should be attributed to HeI (see their Figure 7), our fitting of the spectrum shows that this feature of SN 2017ein can be produced without adding a contribution from He (see Figure 11).

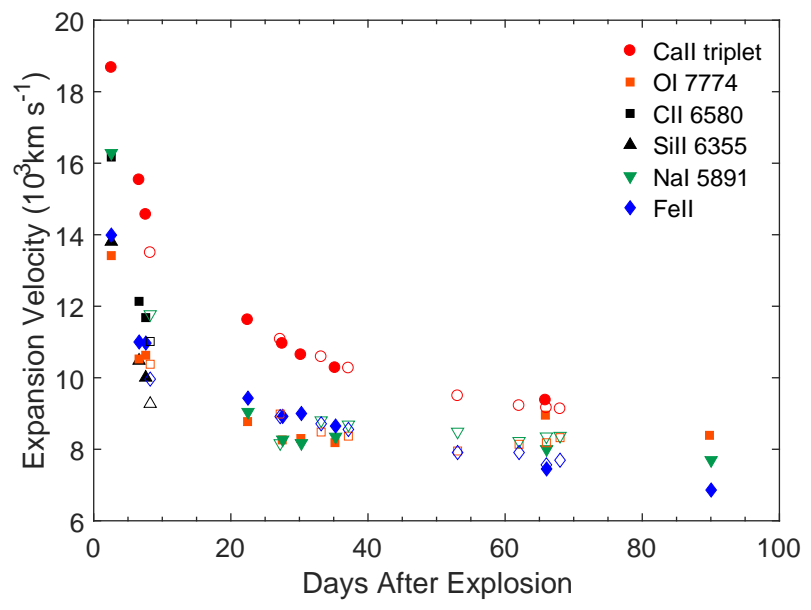

Figure 13. Expansion velocity inferred from absorption lines in the spectra of SN 2017ein. The open symbols represent the data measured from the spectra in Van Dyk et al. (2018).

\section{SHOCK BREAKOUT COOLING}

In this section, we perform a quantitative analysis of the overall evolution of the light curves to investigate the explosion parameters of the supernova and properties of its progenitor. The observation data include the $B V R$-bands light curve from AZT, Swift UVOT, and TNT, and the earliest detection from ATLAS in $c$-band. The light curves of SNe Ic are usually believed to be powered by radioactive decay of ${ }^{56} \mathrm{Ni}$ and ${ }^{56} \mathrm{Co}$ (Arnett 1982), though the magnetar model has recently been proposed to account for some subclasses of
SNe Ic such as SNe Ic-BL (e.g. Wang et al. 2017, 2016; Kasen \& Bildsten 2010) and super luminous supernovae (SLSNe). We note the possible excess emission compared to the power-law fit in the early light curves of SN 2017ein (see Section 3.2), and the similar color evolution with SN 2008D during the first one or two days after explosion (see Figure 5). Given the short duration of these features, it is possible that the early light curve of SN 2017ein is related to the cooling of the shock breakout. So we model the light curves by including a component due to shock cooling.

During the cooling phase, the light curve is dominated by cooling emission from shock breakout (Piro 2015; Piro \& Nakar 2013). This shock cooling model considers a thermodynamical solution of the variation of the luminosity, which is related to energy, mass, and radius of the surrounding material, and expanding velocity of the shocked envelope. The analytical model given by Arnett (1982) can be utilized to compute the bolometric luminosity. Due to the lack of good UV and IR light curve samples, we try to fit the multiband light curves instead of bolometric light curve. To fit the multiband data, we assume a blackbody SED. Combined with the calculated photospheric radius, the effective temperature of the photosphere can be determined, by which the flux density at every band can be calculated. Then we convert the flux density into absolute magnitude.

Based on the Arnett ${ }^{56} \mathrm{Ni}$-decay and shock cooling models and methods described above, we applied the MCMC method (Wang et al. 2017) to fit the observed $B V R$ multi-band light curves of SN 2017ein. The observational data include Swift UVOT, AZT, and TNT light curves, and the magnitudes derived from the first spectrum. We also convert the earliest ATLAS- $c$ photometry to the corresponding value in the $V$-band and include it in the $V$-band light curve. Before that, the five earliest ATLAS- $c$ band data were averaged as they were taken within 1-2 hours. To transform the $c$-band magnitude to the $V$-band, we use the $c-V$ color relationship described in Section 3.2. At the epoch when ATLAS- $c$ photometry was taken, the $c-V$ value is estimated to be $0.14 \pm 0.04 \mathrm{mag}$, from a polynomial fit to the $c-V$ evolution shown in Figure 7 .

In the fitting, the opacity to the ${ }^{56} \mathrm{Ni}$ and ${ }^{56} \mathrm{Co}$ decay photons is adopted as $\kappa_{\gamma, \mathrm{Ni}}=0.027 \mathrm{~cm}^{2} \mathrm{~g}^{-1}$, $\kappa_{\mathrm{opt}}=0.1 \mathrm{~cm}^{2} \mathrm{~g}^{-1}$, respectively, which are typical values for normal SNe Ic. The fitting result including only the contribution of ${ }^{56} \mathrm{Ni}$ decay is shown by the dotted lines in Figure 15, and one can see that it can not explain the early "bump" in the light curves despite that it fits almost the whole light curve in $V$ - and $R$-bands. By fitting the light curves we also find that the pho- 

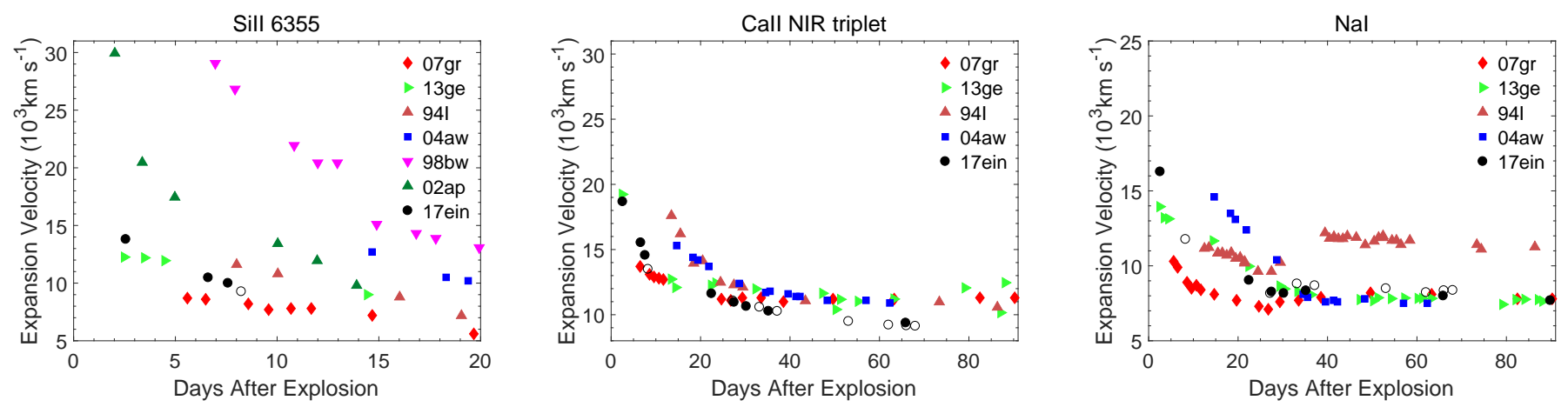

Figure 14. Evolution of the ejecta velocity inferred from SiII $\lambda 6355$ (left), CaII NIR triplet $\lambda 8571$ (middle), and NaID $\lambda 5891$ (right) in the spectra of SN 2017ein and some well-observed SNe Ic. The open circles represent the measurement for SN 2017ein using spectra from Van Dyk et al. (2018).

tosphere cooled to a constant effective temperature of $3000 \sim 4000 \mathrm{~K}$ since $\mathrm{t} \sim 40$ days after explosion. To account for this phenomenon, we apply a technique similar to Nicholl et al. (2017) (see their Equation 9) so that we can also fit the late-time light curve.

The best-fit light curves are shown in Figure 15, and the resultant parameters of the supernova and the progenitor star are reported in Table 4 . We do not show the $I$-band light curve because it can not be fit very well, possibly due to a larger discrepancy between the blackbody assumption and the actual SED of the supernova in the $I$ band, where the spectra of SN are dominated with strong $\mathrm{O}$ and $\mathrm{Ca}$ absorption lines. The fitted parameter $M_{\text {env }}$ is the mass of the outermost shell of the star, from the stellar surface to position where the optical depth is $\sim v / c$ ( $v$ is the shock velocity and $c$ the light speed). Note that $M_{\mathrm{ej}}$ is the rest of the ejected mass of the explosion, while the total ejecta mass is $M=M_{\mathrm{env}}+M_{\mathrm{ej}}$. So the total ejecta mass is $0.92 \pm 0.10 \mathrm{M}_{\odot}$. Our fitting gives the explosion time as MJD 57897.6 \pm 0.6 , which is consistent with that derived in Section 3.2. We thus adopt the explosion time as the weighted average value of these two estimates, i.e. MJD 57897.0 \pm 0.3 .

As shown in Figure 15, the early-time $V$ and $R$-band light curves of SN 2017ein can not be explained by ${ }^{56} \mathrm{Ni}$ heating alone. Adding the contribution from shock cooling improves the fit significantly. The first spectrum was taken at the end of the cooling tail. While the shockcooling feature in the $B$-band is not so convincing, because there are no observations at earlier phases in this waveband when shock-cooling emission was stronger. The emission of the cooling component rises to a peak at about 0.7 day after explosion, and then fades away $\sim 3$ days after explosion. After the cooling phase, the Ni-decay model well reproduces the observations. The model fit with shock cooling gives a stellar envelope radius as $R_{\text {env }}=8 \pm 4 \mathrm{R}_{\odot}$, which is consistent with the radius inferred from analysis of the pre-explosion images (see Section 7). This also suggests that the cooling is from the stellar envelope but not the CSM around the progenitor star. In the latter case, the distance to the outer region of the CSM should be much larger than a few $\mathrm{R}_{\odot}$, and the cooling process will have a longer timescale and produce a more luminous cooling tail. Thus, the early excess emission detected in SN 2017ein is more likely related to shock breakout at the surface of the progenitor star.

Although the "bump" feature in the early light curve seems to be well fit by including a shock cooling contribution, it is still possible that the excess emission was caused by other processes. One popular interpretation is the mixing of ${ }^{56} \mathrm{Ni}$ in the outer layers of the SN ejecta, which is successful in modeling the light curves of some hypernovae like SN 1997ef (Iwamoto et al. 2000), SN 1998bw (Iwamoto et al. 1998; Nakamura et al. 2001), and normal type Ic SN 2013ge (Drout et al. 2016) and iPTF15dtg (Taddia et al. 2016). Mixing of nickel into the outer layers of SN ejecta could produce a fast rise and an extra peak or "bump" in the early phases, as well as broader shaped light curves. However, the nickelmixing model usually has a longer timescale. For example, SN 2013ge has an extra "bump" peaking at about four days after explosion in the UV bands (Drout et al. 2016). While in SN 2017ein, the early-time flux peak appears within one day after explosion, suggestive of a lower possibility for nickel mixing on the surface of the exploding star. Recently Yoon et al. (2018) discussed the effect of Ni-mixing on the early-time photometric evolution of $\mathrm{SNe} \mathrm{Ib} / \mathrm{c}$, and found that the color curves will become progressively red rather than showing an early peak as seen in SN 2008D and SN 2017ein (see Figure 5). We thus prefer the shock cooling model instead of nickel mixing for SN 2017ein.

The ejecta mass we estimated here as $M \sim 1 \mathrm{M}_{\odot}$ is at the lower end of SNe Ib/c (Lyman et al. 2016). For comparison, the ejecta mass of SN 2007gr has been es- 
Table 4. The explosion and progenitor parameters determined from the MCMC fit to the observed $B V R$ light curves of SN 2017ein.

\begin{tabular}{ccccccc}
\hline \hline$M_{\mathrm{ej}}$ & $v_{\mathrm{ph}}$ & $E_{K}$ & $M_{\mathrm{Ni}}$ & $t_{\exp }$ & $R_{\text {env }}$ & $M_{\mathrm{env}}$ \\
$\mathrm{M}_{\odot}$ & $\mathrm{km} \mathrm{s}^{-1}$ & $10^{51} \mathrm{erg}$ & $\mathrm{M}_{\odot}$ & $\mathrm{MJD}$ & $\mathrm{R}_{\odot}$ & $\mathrm{M}_{\odot}$ \\
\hline $0.9 \pm 0.1$ & $9300 \pm 120$ & $\sim 0.5$ & $0.13 \pm 0.01$ & $57897.6 \pm 0.6$ & $8 \pm 4$ & $0.020 \pm 0.004$ \\
\hline
\end{tabular}

timated in the literature, i.e., $2.0-3.5 \mathrm{M}_{\odot}$ by Hunter et al. (2009) and $1.8_{-0.4}^{+0.6} \mathrm{M}_{\odot}$ by Lyman et al. (2016). Hunter et al. (2009) did not describe the details of their fitting. While Lyman et al. (2016) adopts an opacity of $\kappa_{\text {opt }}=0.06 \mathrm{~cm}^{2} \mathrm{~g}^{-1}$ in their analysis, which is smaller than the value adopted here. In the photospheric phase, the Arnett light curve model is described by two parameters: $M_{\mathrm{Ni}}$ and $\tau_{m}$, the latter is given by $\tau_{m}^{2}=\frac{2 \kappa_{\mathrm{opt}} M}{\beta c v_{\mathrm{sc}}}$, where $\beta=13.8$ is a constant, $M$ is the total ejecta mass, $v_{\mathrm{sc}}$ is the scale velocity of the $\mathrm{SN}$, which is observationally set as the photospheric velocity at maximum light $\left(v_{\mathrm{ph}}\right)$. In our model, $v_{\mathrm{ph}}$ is given by the fit with input of the Fe II line velocities measured from the spectra. Hunter et al. (2009) found $v_{\mathrm{ph}} \approx 6,700 \mathrm{~km} \mathrm{~s}^{-1}$ from Si II line, but Lyman et al. (2016) uses $v_{\mathrm{ph}} \approx 10,000 \mathrm{~km} \mathrm{~s}^{-1}$ from Fe II line. The velocity evolution of SN $2007 \mathrm{gr}$ and SN 2017ein shows that the velocity used by Lyman et al. (2016) may be overestimated (see Figure 14). Using the results from Lyman et al. (2016), we find $\tau_{m} \approx 12 \mathrm{~d}$. And we obtain a similar value for SN 2017ein. Therefore, the different $M_{\mathrm{ej}}$ values inferred for SN 2007gr and SN 2017ein are mainly a result of different opacity values adopted in the analysis. Moreover, the discrepancy in the ejected mass could also be related to the fitting methods and wavebands of the light curve used in the analysis.

SN 2017ein is one of the two SNe Ic showing possible evidence for early shock breakout cooling. Another candidate is supernova LSQ14efd, with its $V$-band light curve showing a bump feature at early times, which was also interpreted as cooling of the shocked stellar envelope (Barbarino et al. 2017). In comparison with SN 2017ein, LSQ14efd is more luminous and energetic, with an $R$-band peak absolute magnitude of -18.14 mag, kinematic energy $E_{\text {kin }}=5.3 \mathrm{~B}$, and ejecta mass $M_{\mathrm{ej}}=6.3 \mathrm{M}_{\odot}$, respectively, making it stand between normal SNe Ic and SNe Ic-BL.

\section{HST PROGENITOR OBSERVATION}

7.1. Pre- and post-explosion HST images and data reduction
We searched the pre-explosion HST images from Mikulski Archive for Space Telescopes $\left(\mathrm{MAST}^{6}{ }^{6}\right.$ and the Hubble Legacy Archive (HLA) ${ }^{7}$, and found publicly available $\mathrm{F} 555 \mathrm{~W}$ and $\mathrm{F} 814 \mathrm{~W}$ WFPC2 images taken on 2007 Dec. 11 (PI: W. Li). A F438W-band image at the location of SN 2017ein was taken by HST on 2017 Jun. 12 with the WFC3/UVIS configuration (PI: van Dyk). This image was released and publicly available immediately after the observation. We use this image as a post-explosion image to identify the position of the SN progenitor. Table 5 lists the information of these images.

The pre- and post-discovery HST images around the SN position are shown in Figure 16, where the zoomed-in regions centered at the $\mathrm{SN}$ site are also shown. There is clearly a point-like source near the SN position in both the F555W and F814W images. The position of this point-like source is obtained by averaging the measured values with four different methods (centroid, Gauss, ofilter center algorithm of IRAF task phot, and IRAF task Daophot). On all pre-explosion images, the separation between the position transformed from the postexplosion SN site and the center of the nearby pointlike source is within the uncertainty of the transformed position, suggesting that this nearby point source is the progenitor of SN 2017ein. The coordinates of the SN on the post-explosion F438 image are found to be $(539.302 \pm 0.002,564.516 \pm 0.003)$ with different methods (centroid, Gauss and o-filter center algorithm of IRAF task phot, IRAF task imexamine, and SExtractor). And are converted to $(798.70,1766.77)$ and $(798.38,1766.67)$ on the pre-explosion F555W and F814W images, respectively, as the coordinates of the progenitor.

To get accurate positions of SN 2017ein on the prediscovery images, we first chose several stars that appeared on all the post- and pre-explosion images and then got their positions on each image using SExtractor. A second-order polynomial geometric transformation function is applied using the IRAF geomap task to

\footnotetext{
6 http://archive.stsci.edu/

7 http://hla.stsci.edu/
} 

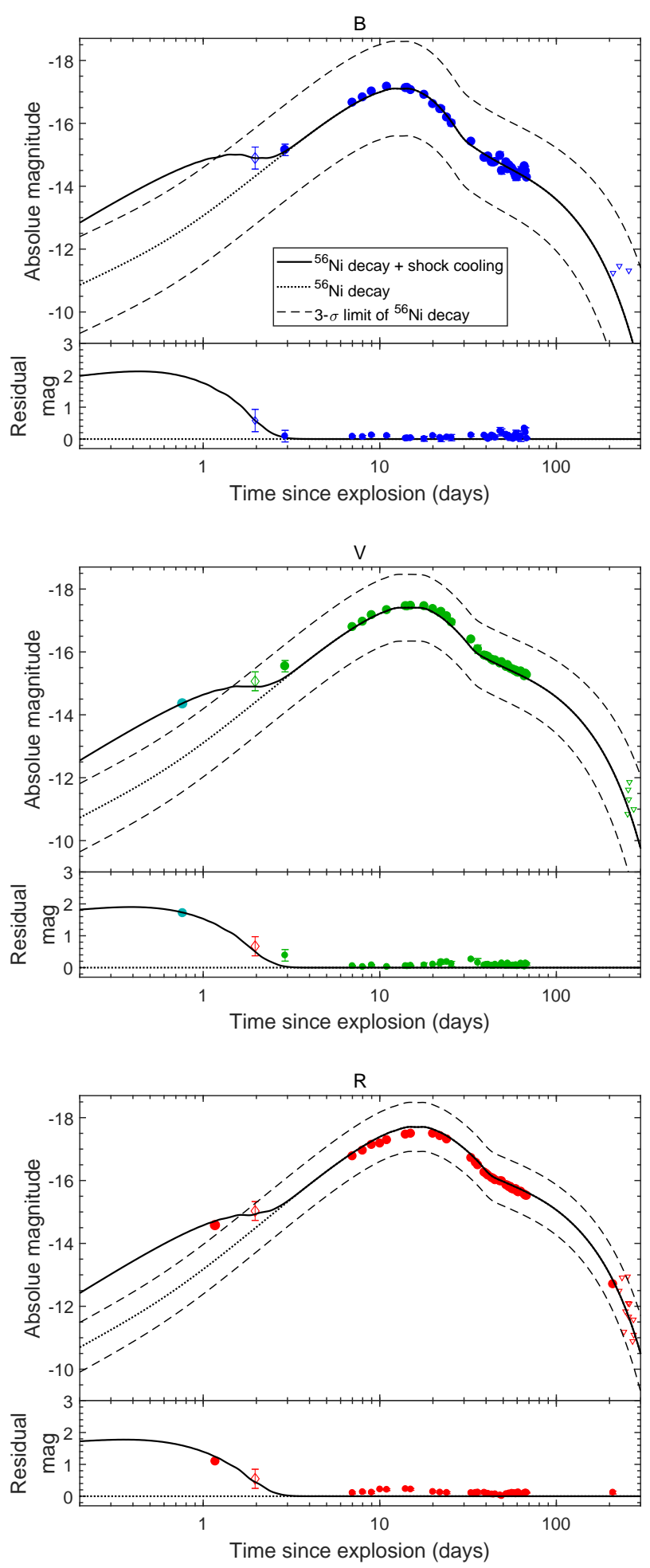

Figure 15. The fitting results of the $B V R$ light curves of SN 2017ein. Each subplot shows one band. The absolute magnitudes from ATLAS, Swift UVOT, AZT and TNT data are shown by solid symbols. The open diamond represents the magnitude derived from the first spectrum taken on 2017 May 26.57. The data point in cyan represent the magnitude converted from ATLAS $c$-band magnitudes in the $V$-band figure (see text for details). The lower panel of each plot shows the residual of the shock cooling model (solid line) and observations (data points) relative to non-cooling model. convert their coordinates on the post-explosion image to those on the pre-explosion images. Based on the IRAF geoxytran task, this established the transformation relationship between the coordinates of SN 2017ein on the post-explosion image and those on the preexplosion images. The uncertainties of the transformed coordinates are a combination of the uncertainties in the SN position and the geometric transformation. The transformed SN and progenitor positions and their uncertainties are listed in Table 6. As one can see, the location of the SN center and the point-like source on the pre-explosion F555W and F814W images have small offsets of $0^{\prime \prime} .020$ and $0^{\prime \prime} .030$, respectively, which are smaller than the uncertainty of the location of the supernova.

We use DOLPHOT ${ }^{8}$ to get photometry of the progenitor on the pre-explosion images. The photometry is performed on raw C0M FITS images obtained from the MAST archive. Magnitudes and their uncertainties of the progenitor candidate are extracted from the output of DOLPHOT. We find that the progenitor star has magnitudes of $24.46 \pm 0.11 \mathrm{mag}$ and $24.39 \pm 0.17 \mathrm{mag}$ in F555W band and F814W band, respectively. Note that our measurements are brighter by about $0.1 \mathrm{mag}$ in $\mathrm{F} 555 \mathrm{~W}$ and $0.2 \mathrm{mag}$ in $\mathrm{F} 814 \mathrm{~W}$, respectively, compared with the results recently reported by Van Dyk et al. (2018).

To further constrain the properties of the identified point-like source at the SN position, we also studied the photometric properties of the surrounding stars within 5 arc-second, corresponding to a radius of about 400 pc, around the progenitor. The measured PSF FWHMs of these stars are in a range of $0^{\prime \prime} .15 \sim 0^{\prime \prime} .20$ in both F555W and F814W bands, which are consistent with that derived for the progenitor (i.e. $0^{\prime \prime} .21$ in F814W). While these FWHMs of the stars correspond to a radius of $\sim 16 \mathrm{pc}$, which is possible for a compact star cluster. So we can not be sure about whether the progenitor candidate is a single star, a multi-system, or a star cluster.

\subsection{Progenitor properties: a single WR star?}

From the HST photometry and the reddening derived in Section 4, we found that the progenitor star has a very high luminosity with $\mathrm{M}_{\mathrm{F} 555 \mathrm{~W}}=-8.17 \pm 0.37 \mathrm{mag}$ and a very blue color of $V-I=-0.47 \pm 0.27 \mathrm{mag}$. This indicates that SN 2017ein may have had a blue and luminous progenitor star. Despite the complexity of the progenitor candidate, for simplicity, we first consider it as a single star.

Metallicity is another important parameter to constrain the properties of the progenitor star. From the

\footnotetext{
${ }^{8}$ http://americano.dolphinsim.com/dolphot/
} 

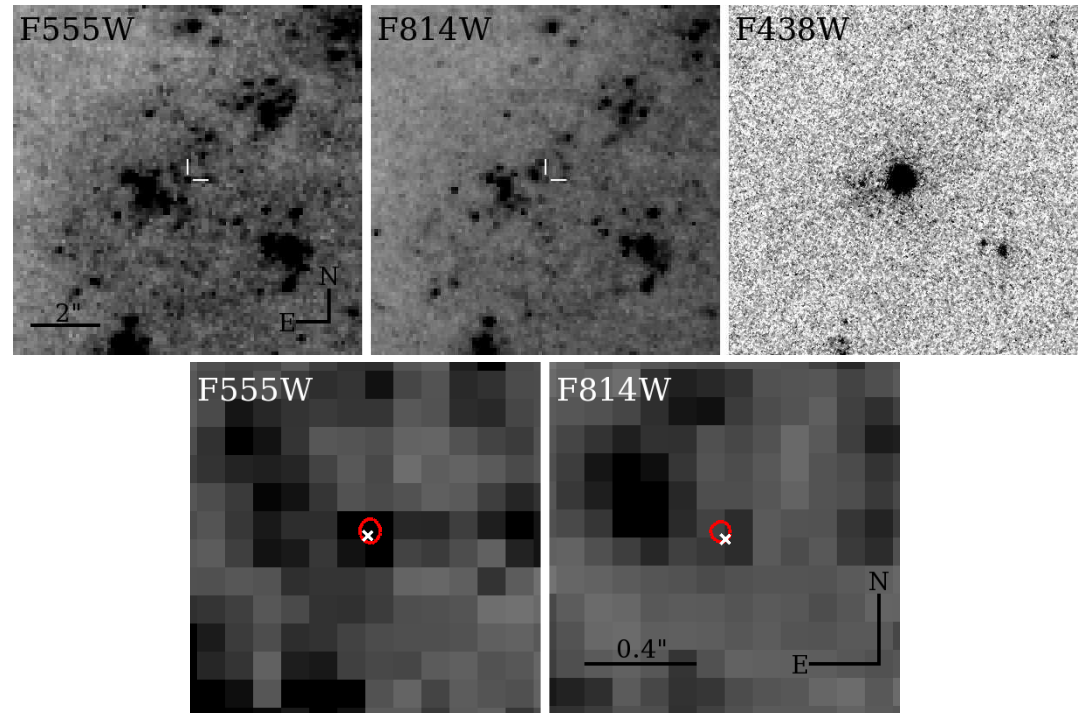

Figure 16. Upper panel: The regions around SN 2017ein showing on the pre-discovery HST F555W (left) and F814W (middle) images, and on the post-explosion HST F438W image (right). The white ticks mark the location of the progenitor star. Lower panel: the zoomed-in pre-explosion HST images centering around the progenitor candidate in F555W (left) and F814W (right) filters, with the white crosses marking the center positions of the identified progenitor star. The center of red ellipses shows the SN position, with the radius of the ellipse showing the size of the error. All images are aligned.

Table 5. Pre- and post-explosion HST images on the site of SN 2017ein.

\begin{tabular}{ccccccc}
\hline \hline Instrument & Filter & Obs. date & Exposure time(s) & Proposal ID & PI & Drizzled image \\
\hline WFPC2 & F555W & $2007-12-11$ & $230 \times 2$ & 10877 & Li & hst_10877_10_wfpc2_f555w_wf_drz.fits \\
WFPC2 & F814W & $2007-12-11$ & $350 \times 2$ & 10877 & Li & hst_10877_10_wfpc2_f814w_wf_drz.fits \\
WFC3/UVIS & F438W & $2017-06-12$ & $10 \times 27$ & 14645 & van Dyk & id9703010_drc.fits \\
\hline
\end{tabular}

measurements of Kaplan et al. (2016), the metallicity at the site of SN 2017ein is found to be $\log \left(Z / Z_{\odot}\right) \approx 0.22$ or $12+\log (\mathrm{O} / \mathrm{H}) \approx 8.91^{9}$. Such high metallicity for the progenitor is consistent with the general trend that normal SNe Ic tend to be metal rich compared to the subclass of SNe Ic-BL or GRB-SNe (Heger et al. 2003). Considering the high luminosity and blue color, we assume that it is a WR star. The lack of $\mathrm{H}$ features in the supernova spectra indicates a $\mathrm{H}$ poor progenitor. Radiative transfer models including non-local thermodynamical equilibrium effects of SE SNe (see Hachinger et al. 2012, and references therein) suggest that only $\sim 0.1 \mathrm{M}_{\odot}$ of helium is needed in the progenitor star to produce an observable signature in the spectra. As we do not see any significant helium features in SN 2017ein, its progenitor must also be helium poor. Thus, we use

\footnotetext{
9 We adopt the average value of metallicity at the SN location measured by various methods in the literature (Kaplan et al. 2016), and the solar metallicity as $12+\log (\mathrm{O} / \mathrm{H})=8.69$ (Asplund et al. 2009).
}

the model spectra grids of Milky Way-like WC-type WR stars (Sander et al. 2012) to fit the photometry data of the progenitor star. The model spectra are characterized by two parameters: the effective temperature $T_{\text {eff }}$ and the "transformed radius" $R_{t}$, which is defined by

$$
R_{t}=R_{*}\left[\frac{v_{\infty}}{\mathrm{km} \mathrm{s}^{-1}} / \frac{\dot{M} \sqrt{D}}{10^{-4} M_{\odot} \mathrm{yr}^{-1}}\right]^{2 / 3}
$$

Where $\dot{M}$ is the mass loss rate of the star, $v_{\infty}$ is the terminal wind velocity $\left(v_{\infty}=2000 \mathrm{~km} \mathrm{~s}^{-1}\right.$ for WC stars), and $D$ is the so-called density contrast, which is set to be 10 for WC stars. The flux of model spectra is normalized so that the luminosity of the star is $10^{5.3}$ times that of the sun.

Due to the fact that we only have observation in two bands, we use a MC simulation to better estimate the range of the stellar parameters. We created a sample of fluxes in F555W and F814W bands using the measured magnitudes and extinction to the SN. Then we fit the color index F555W-F814W to that of the model spectra to determine the effective temperature. The radius 
Table 6. Transformed SN positions on pre-explosion HST images

\begin{tabular}{ccc}
\hline \hline image & F555W & F814W \\
\hline$s_{\text {pre }} /\left(\text { arcsec } \cdot \text { pixel }^{-1}\right)^{\mathrm{a}}$ & 0.10 & 0.10 \\
$N_{\text {stars }}$ & 14 & 16 \\
$\sigma_{\text {geomap }} /$ pixel $^{\mathrm{c}}$ & $0.36 / 0.43$ & $0.34 / 0.36$ \\
$\sigma_{\text {geomap }} /$ mas $^{\mathrm{d}}$ & $36 / 43$ & $34 / 36$ \\
$s_{\text {post }} /\left(\text { arcsec } \cdot \text { pixel }^{-1}\right)^{\mathrm{e}}$ & & 0.04 \\
$\sigma_{\mathrm{SN}} / \mathrm{mas}^{\mathrm{f}}$ & \multicolumn{3}{c}{$0.1 / 0.1$} \\
$\sigma_{\text {total }} / \mathrm{mas}^{\mathrm{g}}$ & $35.8 / 42.7$ & $34.1 / 35.8$ \\
$(x, y)_{\mathrm{SN}^{\mathrm{h}}}$ & $(798.70,1766.77)$ & $(798.38,1766.67)$ \\
$(x, y)^{* \mathrm{i}}$ & $(798.60,1766.60)$ & $(798.56,1766.43)$ \\
$\sigma_{*} / \mathrm{mas}^{\mathrm{j}}$ & $9 / 5$ & $17 / 7$ \\
$d / \mathrm{mas}^{\mathrm{k}}$ & 20 & 30 \\
\hline
\end{tabular}

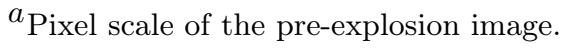

${ }^{b}$ Number of stars used in the geometric transformation.

${ }^{c}$ Uncertainty of the geometric transformation in pixel.

$d_{\text {Uncertainty of the geometric transformation in milliarcseconds. }}$

${ }^{e}$ Pixel scale of the post-explosion image.

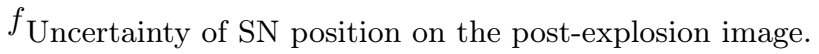

$g_{\text {Uncertainty of the transformed SN position on pre-explosion im- }}$ age.

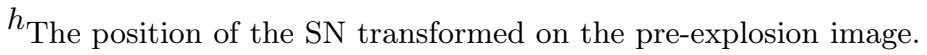

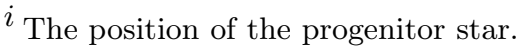

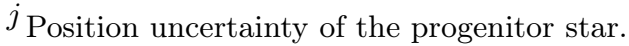

${ }^{k}$ Separation of the SN from the progenitor star.

and luminosity of the star are then determined using the scale factor between the apparent flux in $\mathrm{F} 555 \mathrm{~W}$ and F814W bands and that of the model SED. Finally, we get distributions of effective temperature, luminosity and radius of the progenitor candidate. By taking the full-width at half maximum (FWHM) of the distributions of the parameters, this analysis gives estimates of an effective temperature range of $\sim 70,800-15,900 \mathrm{~K}$, a luminosity of $6.1 \lesssim \log L / L_{\text {odot }} \lesssim 7.3$, and a stellar radius of $6 \sim 14 \mathrm{R}_{\odot}$. We caution, however, that current estimates of the progenitor parameters contain considerable uncertainties due to large errors associated with the photometry, distance, and extinction of the progenitor star. Moreover, for WR stars with very high temperature, the radiation in the optical is only a small proportion of its total luminosity (Crowther 2007), and this will also give rise to a large uncertainty in determining the temperature from only two optical bands like F555W and F814W.

Figure 17 shows the stellar evolution tracks of massive stars at solar metallicity (Georgy et al. 2012), with the locus of the progenitor of SN 2017ein overplotted. These evolution models take rotational effects into account, with an initial rate of $v_{\text {ini }} / v_{\text {crit }}=0.4$ (see also the model descriptions in Ekström et al. 2012). Different colors of tracks indicate different stellar types, which are determined following the definitions proposed by Meynet \& Maeder (2003) and Smith \& Maeder (1991). Inspecting Figure 17, we propose that the initial mass of the progenitor star of SN 2017ein was larger than $60 \mathrm{M}_{\odot}$. According to these stellar evolution models, the final CO core mass can be $\sim 17.5 \mathrm{M}_{\odot}$ for massive stars with initial mass of $60 \mathrm{M}_{\odot}$, and the explosion as SN Ic can produce an ejecta mass of $>10 \mathrm{M}_{\odot}$, with a black hole remnant. This expected ejecta mass is rather large compared to the value we inferred for SN 2017ein. However, this mass estimate may be too large, although the progenitor radius that we get from the SED fitting is not far from the value given by the shock cooling fitting. Given the uncertainty in deriving the bolometric luminosity, it is likely that the progenitor luminosity is somewhat overestimated due to the lack of multiband photometry data especially at shorter wavelengths.

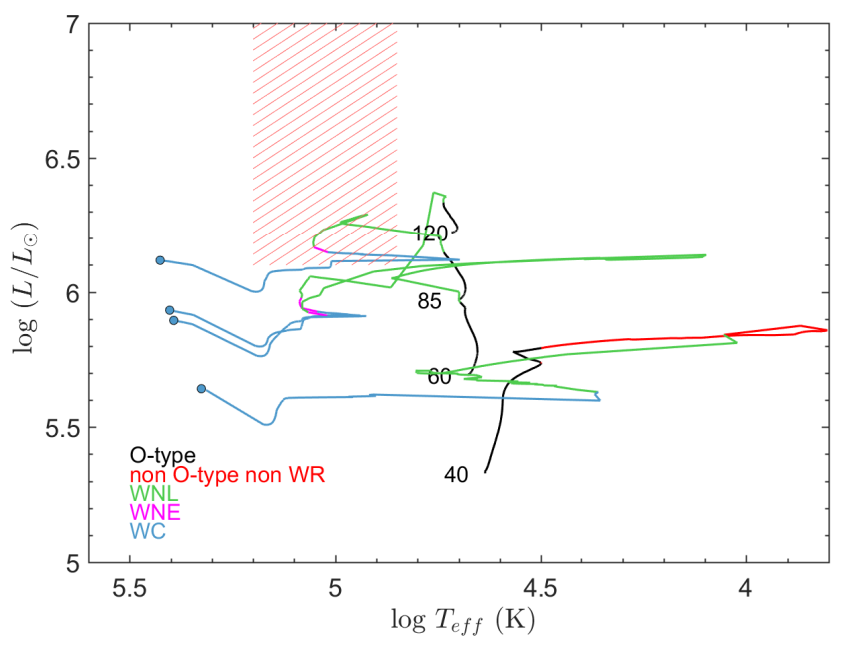

Figure 17. Hertzsprung-Russel diagram showing the locus of the progenitor of SN 2017ein. The evolutionary tracks of massive stars at solar metallicity from Georgy et al. (2012) are displayed for comparison. The numbers in the plot mark the corresponding ZAMS mass in unit of solar mass. The endpoints of the tracks are indicated by dots. Different colors on the lines represent different evolutionary stages of the stars.

7.3. Binary, star cluster and low ejecta mass 
As an alternative, the high luminosity of the progenitor may be related to the contribution of a similarly luminous companion star. According to observations of WR stars in the Milky Way and Large Magellanic Cloud (LMC), one finds that more than $80 \%$ of luminous stars, i.e., $\mathrm{M}_{V}<-5.5 \mathrm{mag}$, are found to be binaries, while this fraction drops to about $50 \%$ for the relatively faint counterparts (Hainich et al. 2014; van der Hucht 2006). After examining the surrounding stars within a radius of 5 arc-seconds from the progenitor of SN 2017ein on the pre-explosion images, we find that the progenitor star does not stand out from them but clearly belongs to the relatively blue and luminous subgroup before subtracting host extinction, as shown in Figure 18. This, perhaps, favors a multi-system or star cluster as the source. In this case, we expect to see a bright star at the SN position when the supernova fades away in a few years.

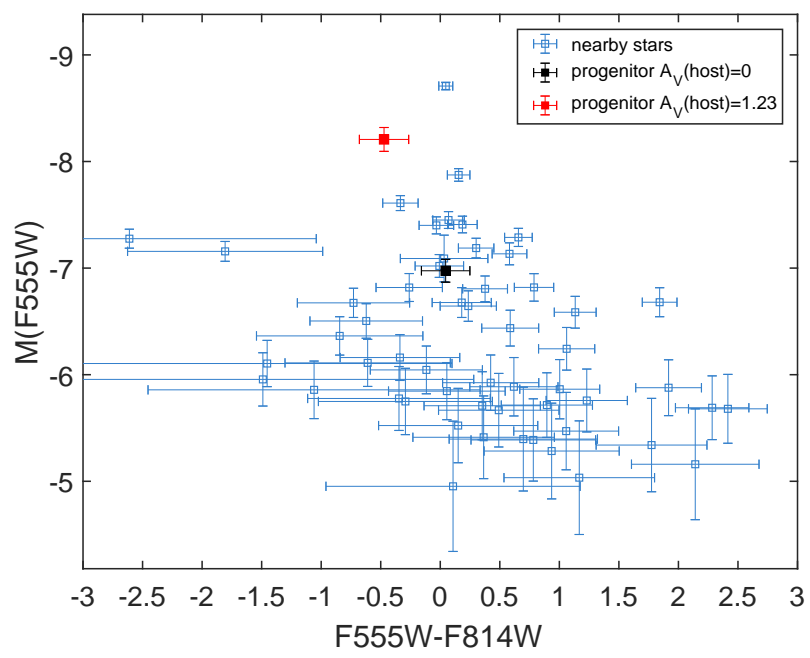

Figure 18. A color-absolute magnitude diagram of the progenitor candidate of SN 2017ein and its surrounding stars at a distance within 5 arcseconds. The absolute magnitudes and color index of the surrounding stars are corrected for Galactic extinction but not the host extinction. A distance modulus of $\mu=31.38 \mathrm{mag}$ is adopted.

Binary interaction can result in more efficient mass loss for massive stars, resulting in lower ejecta masses in supernovae. Lyman et al. (2016) found that explosions of stars with initial mass larger than $28 \mathrm{M}_{\odot}$ will produce ejecta of mass not lower than $5 \mathrm{M}_{\odot}$, and even stars with initial mass $\geqslant 20 \mathrm{M}_{\odot}$ in binaries will produce ejecta of mass higher than $5 \mathrm{M}_{\odot}$. Thus, lower-mass binary models are in better agreement with the distribution of ejecta mass of $\mathrm{SNe} \mathrm{Ib} / \mathrm{c}$. Considering the lower ejecta mass of $\sim 1 \mathrm{M}_{\odot}$ for SN 2017ein, it is more likely that its progenitor star is a lower mass star in a binary system rather than a single star with such a high initial mass. As discussed in Van Dyk et al. (2018), the progenitor is consistent with massive binary stars. Kilpatrick et al. (2018) has came to similar conclusions, prefering a binary model of $80+48 \mathrm{M}_{\odot}$. However, the expected ejecta mass is still higher than $5 \mathrm{M}_{\odot}$.

These results have demonstrated a connection between SN 2017ein and very massive progenitor stars, conflicting with the low ejecta mass. This may be related to mass loss in massive stars. The mass loss of massive stars dominates their evolution and determines their fate (Smith 2014). However, the mass loss of massive stars is quite complicated. In addition to mass loss through strong and relatively mild stellar wind as WR stars, massive stars may experience eruptive mass loss, e.g. Luminous Blue Variables (LBVs). And some stars undergo explosive mass loss shortly (a few years) before explosion (Ofek et al. 2014; Gal-Yam et al. 2014; Yaron et al. 2017). Circumstellar dust can give rise to large uncertainty in observations of massive stars. Some WR systems reveal persistent or episodic dust formation within months or years, resulting in strong optical or IR variations with an amplitude of a few magnitudes of the WR binaries, due to the newly formatted dust or the asymmetry of the dust distribution (eg. Williams et al. 2009). This dust will eventually be ejected to the interstellar medium (ISM).

On the other hand, as also proposed by Van Dyk et al. (2018), the supernova explosion can also disrupt and vaporize circumstellar dust. These facts demonstrate that the extinction derived from the $\mathrm{Na}$ I D lines in the SN spectra, which mostly represent extinction from the interstellar dust, rather than the dust surrounding the star, can not be directly applied to the progenitor. The extinction to the SN should be a lower limit of the true extinction to the progenitor. As a result, the progenitor could be even bluer and brighter. Combining the effects of dust formation in massive stars and destruction by the $\mathrm{SN}$ explosion, the real extinction to the progenitor can be very complicated. Detailed discussions on the mass loss and dust formation and disruption in SN 2017ein is beyond the scope of this paper.

On the other hand, the progenitor candidate could also be a young and blue star cluster, as also pointed out by Van Dyk et al. (2018). As noted previously, a $\lesssim 16$ pc cluster would be unresolved in the pre-explosion data. To explore this possibility, we created a set of models for a $10^{4} \mathrm{M}_{\odot}$ cluster with the GALEV code (Kotulla et al. 2009). We assume a single stellar population at solar metallicity, formed in a exponentially declining burst. In Figure 19, we compare the colour and magnitude of such a cluster to the source observed to be coincident with 


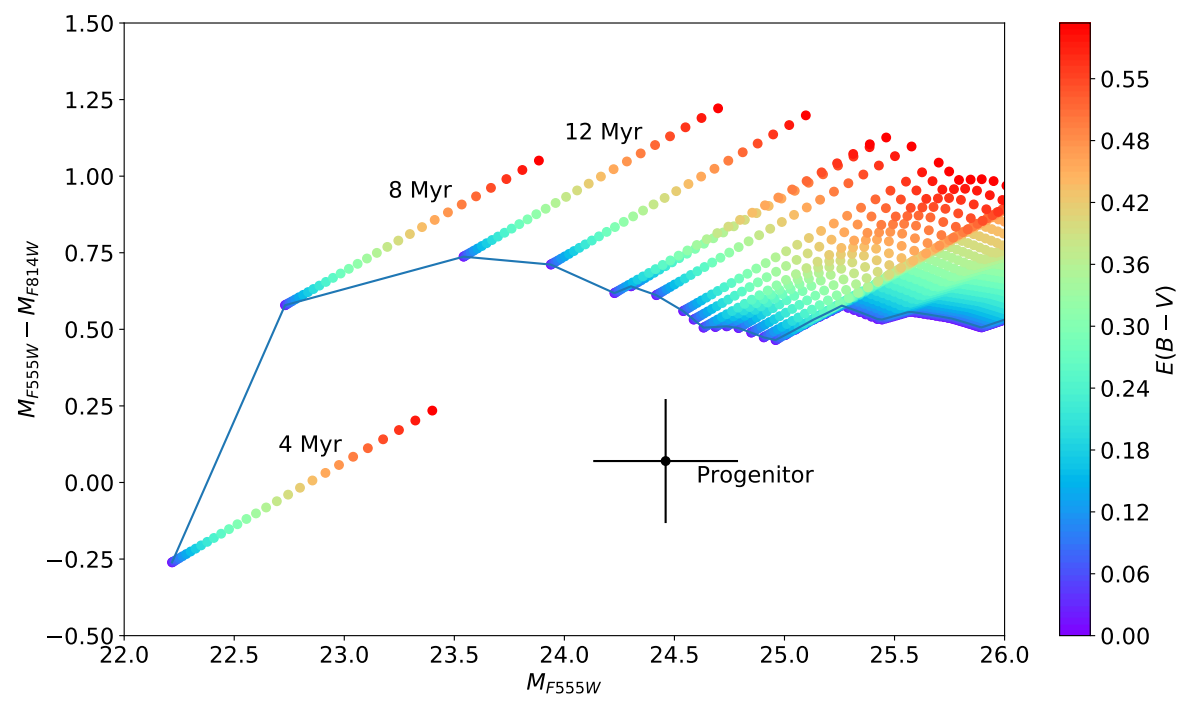

Figure 19. A set of cluster models from GALEV, for a $1 \times 10^{4} \mathrm{M}_{\odot}$ cluster at solar metallicty. The color of each point denotes the assumed total reddening, while the line connects the $E(B-V)=0$ cluster models to guide the eye. All tracks have placed at the distance of SN 2017ein. The observed progenitor color and magnitude is indicated.

the SN. The relatively blue color of the source implies that the progenitor must be $\sim 4$ Myr old. The absolute magnitude of the observed source is much fainter than the model cluster, implying that it must be substantially less massive. Naively scaling our model magnitudes to the observed value, we find that the cluster would have a mass $\sim 2.5 \times 10^{3} \mathrm{M}_{\odot}$. As a test of our results, we also compared to the colours of a set of BPASS models (v 2.2.1, Stanway \& Eldridge 2018), and found similar results.

Such a young cluster age implies that in this case the progenitor would have to be relatively massive. Taking the lifetimes of single massive stars from the STARS code (Eldridge et al. 2008), we find that the progenitor would of necessity have a zero age main sequence mass of $\gtrsim 40 \mathrm{M}_{\odot}$. Such a star would in fact lose its $\mathrm{H}$ envelope through stellar winds, and explode as a single WR star. For a Salpeter initial mass function between 0.1 and $300 \mathrm{M}_{\odot}$, and assuming a cluster of $2500 \mathrm{M}_{\odot}$, then only around $160 \mathrm{M}_{\odot}$ of the cluster mass will be in stars more massive than $40 \mathrm{M}_{\odot}$. So, if this were indeed the case, then the progenitor was one of the most massive members of the cluster.

The preceding analysis must be taken with considerable caution: with only two broadband filters it is extremely difficult to draw firm conclusions as to the properties of any possible cluster, especially when young clusters of hot massive stars will emit much of their flux at bluer wavelengths than F555W. We also caution that we cannot even be certain as to whether we are observ- ing a single population - even a small $16 \mathrm{pc}$ region could plausibly play host to multiple overlapping stellar populations. Future observations, and in particular in the $\mathrm{UV}$, can help resolve this uncertainty.

\section{CONCLUSIONS}

We present the optical/ultraviolet observations of the nearby type Ic supernova SN 2017ein and analysis of the properties of the supernova and its progenitor, by taking advantages of both our extremely early-time photometry/spectroscopy and the public HST pre-explosion data. SN 2017ein is similar to SN 2007gr and SN 2013ge in overall spectral evolution, all characterized by prominent carbon features in the early phase, while SN 2017ein clearly shows some distinct features from the latter two. The expansion velocities of different lines of SN 2017ein are similar to the other two, but the Na I and Si II expansion velocities of SN 2017ein are higher than those of SN 2007gr, but comparable to those of SN 2013ge. Another interesting feature is that the absorption lines of the CaII NIR triplet split into two narrow lines at late phases, which was not seen in other $\mathrm{SNe}$ $\mathrm{Ib} / \mathrm{c}$ including SNe 2007gr and 2013ge. This probably suggests that the line forming region of SN 2017ein is confined into a relatively narrow shell. Moreover, the late time light curves of SN 2017ein are fast declining, which is not seen in other $\mathrm{SNe} \mathrm{Ib} / \mathrm{c}$.

From the very early photometry and non detection limit, we were able to constrain the first-light time of SN 2017ein to be within one day after explosion, rep- 
resenting one of the tightest constraints for $\mathrm{SNe} \mathrm{Ib} / \mathrm{c}$. Most interestingly, the early data show a possible excess emission relative to the $\mathrm{Ni}$ decay model at one day after explosion, which can be attributed to the shock cooling tail. By fitting the $B V R$-band light curves, using a radioactive decay model and a shock cooling model, we find that the supernova synthesized ${ }^{56} \mathrm{Ni}$ mass of $\sim 0.13 \mathrm{M}_{\odot}$, and the stellar envelope has a radius of $8 \pm 4 \mathrm{R}_{\odot}$ and a mass of $\sim 0.02 \mathrm{M}_{\odot}$. Note that a smaller ejecta mass of $\sim 1 \mathrm{M}_{\odot}$ is found for SN 2017ein. This indicates that the progenitor star has lost most of its $\mathrm{H} / \mathrm{He}$ envelope before collapse, consistent with a compact Wolf-Rayet star or a massive binary. SN 2017ein perhaps represents the first normal SN Ic observed to have a signature of shock breakout cooling from the stellar envelope. Undoubtedly, it provides a very good sample to test theories of SN Ic explosions.

By analyzing the pre-explosion HST images, we find a point-like source with an absolute magnitude of $M_{V}=$ $-8.00 \pm 0.34 \mathrm{mag}$ and $M_{I}=-7.53 \pm 0.35 \mathrm{mag}$ coincides with the position of SN 2017ein. Assuming that the source is a single star, it is found to have an effective temperature of $\sim 70,800-159,000 \mathrm{~K}$, a luminosity of $\log \left(L / L_{\odot}\right) \gtrsim 6.1$, and a radius of $\sim 6-14 \mathrm{R}_{\odot}$, roughly consistent with a hot and luminous WR star. Comparing with stellar evolutionary tracks, we found that the initial mass of this star could exceed $60 \mathrm{M}_{\odot}$. Such a hot and luminous star is very rare and we did not find a similar star in current WR star catalogs. However, such massive star is inconsistent with the low ejecta mass found for SN 2017ein. On the other hand, it is also possible that the point source identified at the SN position could be a binary or a young, blue star cluster. The low ejecta mass inferred for SN 2017ein seems to favor for a low mass binary scenario. By comparing the observational data and SSP star cluster models at solar metallicity, we find that the source is consistent with star clusters with age of $4 \mathrm{Myr}$ and a low mass of $\sim 2.5 \times 10^{3} \mathrm{M}_{\odot}$. And the progenitor of SN 2017ein should be among the most massive members of the cluster. Due to the low resolution of the HST images and the lack of data in shorter wavelengths, it is difficult to decide which case is favored. Further distinguishing between different scenarios needs to wait for another visit of this galaxy with HST when the SN light dims out. SN 2017ein provides a rare opportunity that helps to throw light on massive star evolution and supernova explosion theories.

We acknowledge the support of the staff of the Xinglong $2.16 \mathrm{~m}$ and Lijiang $2.4 \mathrm{~m}$ telescope. This work is supported by the National Natural Science Foundation of China (NSFC grants 11178003, 11325313, and 11633002), the National Program on Key Research and Development Project (grant no. 2016YFA0400803), and the Tsinghua University Initiative Scientific Research Program. J.-J. Zhang is supported by the NSFC (grants 11403096, 11773067), the Key Research Program of the CAS (Grant NO. KJZD-EW- M06), the Youth Innovation Promotion Association of the CAS (grants 2018081), and the CAS "Light of West China" Program. T.-M. Zhang is supported by the NSFC (grants 11203034). This work was also partially Supported by the Open Project Program of the Key Laboratory of Optical Astronomy, National Astronomical Observatories, Chinese Academy of Sciences. Funding for the LJT has been provided by Chinese Academy of Sciences and the People's Government of Yunnan Province. The LJT is jointly operated and administrated by Yunnan Observatories and Center for Astronomical Mega-Science, CAS.

We thank the staff of AZT and ATLAS for their observations and allowance of the use of the data. ATLAS is funded by NASA grant NN12AR55G under the Near Earth Objects Observations program (NEOO). L.J.W. acknowledges the support of the National Program on Key Research and Development Project of China (Grant No. 2016YFA0400801). This work makes use of data from the Las Cumbres Observatory network and the Global Supernova Project. DAH, CM, and GH are supported by the US National Science Foundation (NSF) grant AST-1313484. EB and JD are supported in part by NASA Grant NNX16AB256. MF is supported by a Royal Society - Science Foundation Ireland University Research Fellowship. J.V. is supported by the project "Transient Astrophysical Objects" GINOP 2.3.215-2016-00033 of the National Research, Development and Innovation Office (NKFIH), Hungary, funded by the European Union. K. Nomoto, A. Tolstove, and S. Blinnikov are supported by the World Premier International Research Center Initiative (WPI Initiative), MEXT, Japan, and JSPS KAKENHI (grant numbers JP16K17658, JP26400222, JP16H02168, JP17K05382). S.B. acknowledges funding from project RSF 18-1200522 .

Software: ZrutyPhot (Mo et al. in prep.), SYNAPPS (Thomas et al. 2011), IRAF (Tody 1993, 1986), DOLPHOT (Dolphin 2016), SExtractor (Bertin \& Arnouts 1996), GALEV (Kotulla et al. 2009), STARS (Eldridge et al. 2008). 


\section{REFERENCES}

Arcavi, I., Hosseinzadeh, G., Brown, P. J., et al. 2017, ApJL, 837, L2

Arnett, W. D. 1982, Astrophysical Journal, 253, 785

Asplund, M., Grevesse, N., Sauval, A. J., \& Scott, P. 2009, ARA\&A, 47, 481

Barbarino, C., Botticella, M. T., Dall'Ora, M., et al. 2017, MNRAS, 471, 2463

Barbon, R., Benetti, S., Cappellaro, E., et al. 1995, Astronomy and Astrophysics Supplement, 110, 513

Berger, E., Kulkarni, S. R., \& Chevalier, R. A. 2002, ApJL, $577, \mathrm{~L} 5$

Bersten, M. C., Benvenuto, O. G., Folatelli, G., et al. 2014, AJ, 148,68

Bersten, M. C., Folatelli, G., Garcíła, F., et al. 2018, Nature, 554, 497

Bertin, E., \& Arnouts, S. 1996, A\&AS, 117, 393

Bianco, F. B., Modjaz, M., Hicken, M., et al. 2014, The Astrophysical Journal Supplement, 213, 19

Breeveld, A. A., Landsman, W., Holland, S. T., et al. 2011, in American Institute of Physics Conference Series, Vol. 1358, American Institute of Physics Conference Series, ed. J. E. McEnery, J. L. Racusin, \& N. Gehrels, 373-376

Brown, P. J., Breeveld, A. A., Holland, S., Kuin, P., \& Pritchard, T. 2014, Ap\&SS, 354, 89

Campana, S., Mangano, V., Blustin, A. J., et al. 2006, Nature, 442, 1008

Cano, Z. 2014, ApJ, 794, 121

Cao, Y., Kasliwal, M. M., Arcavi, I., et al. 2013, ApJL, 775, L7

Chen, J., Wang, X., Ganeshalingam, M., et al. 2014, Astrophysical Journal, 790, 120

Clocchiatti, A., Wheeler, J. C., Brotherton, M. S., et al. 1996, Astrophysical Journal, 462, 462

Corsi, A., Ofek, E. O., Frail, D. A., et al. 2011, ApJ, 741, 76

Crowther, P. A. 2007, Annual Review of Astronomy \& Astrophysics, 45, 177

Dessart, L., Hillier, D. J., Li, C., \& Woosley, S. 2012, MNRAS, 424, 2139

Dolphin, A. 2016, DOLPHOT: Stellar photometry, Astrophysics Source Code Library, , , ascl:1608.013

Drout, M. R., Soderberg, A. M., Gal-Yam, A., et al. 2011, ApJ, 741, 97

Drout, M. R., Milisavljevic, D., Parrent, J., et al. 2016, Astrophysical Journal, 821, 57

Ehgamberdiev, S. 2018, Nature Astronomy, 2, 349

Ekström, S., Georgy, C., Eggenberger, P., et al. 2012, A\&A, 537, A146

Eldridge, J. J., Fraser, M., Maund, J. R., \& Smartt, S. J. 2015, MNRAS, 446, 2689
Eldridge, J. J., Fraser, M., Smartt, S. J., Maund, J. R., \& Crockett, R. M. 2013, MNRAS, 436, 774

Eldridge, J. J., Izzard, R. G., \& Tout, C. A. 2008, MNRAS, 384,1109

Eldridge, J. J., \& Maund, J. R. 2016, MNRAS, 461, L117

Filippenko, A. V., Barth, A. J., Matheson, T., et al. 1995, Astrophysical Journal, 450, 11

Foley, R. J., Papenkova, M. S., Swift, B. J., et al. 2003, The Publications of the Astronomical Society of the Pacific, 115,1220

Fox, O. D., Azalee Bostroem, K., Van Dyk, S. D., et al. 2014, ApJ, 790, 17

Fremling, C., Sollerman, J., Taddia, F., et al. 2014, A\&A, 565, A 114

Fremling, C., Sollerman, J., Kasliwal, M. M., et al. 2018, A\&A, 618, A37

Frey, L. H., Fryer, C. L., \& Young, P. A. 2013, ApJL, 773, L7

Gal-Yam, A., Ofek, E. O., \& Shemmer, O. 2002, Monthly Notices of the Royal Astronomical Society, 332, L73

Gal-Yam, A., Arcavi, I., Ofek, E. O., et al. 2014, Nature, 509,471

Galama, T. J., Vreeswijk, P. M., van Paradijs, J., et al. 1998, Nature, 395, 670

Gehrels, N., Chincarini, G., Giommi, P., et al. 2004, ApJ, 611,1005

Georgy, C., Ekström, S., Meynet, G., et al. 2012, Astronomy \& Astrophysics, 332, A29

Georgy, C., Ekström, S., Eggenberger, P., et al. 2013, A\&A, 558, A103

Gordon, K. D., Clayton, G. C., Misselt, K. A., Landolt, A. U., \& Wolff, M. J. 2003, ApJ, 594, 279

Groh, J. H., Georgy, C., \& Ekström, S. 2013, A\&A, 558, L1

Guillochon, J., Parrent, J., Kelley, L. Z., \& Margutti, R. 2017, ApJ, 835, 64

Hachinger, S., Mazzali, P. A., Taubenberger, S., et al. 2012, MNRAS, 422, 70

Hainich, R., Rühling, U., Todt, H., et al. 2014, Astronomy \& Astrophysics, 565, A27

Hamuy, M., \& Pinto, P. A. 2002, ApJL, 566, L63

Heger, A., Fryer, C. L., Woosley, S. E., Langer, N., \& Hartmann, D. H. 2003, Astrophysical Journal, 591, 288

Huang, F., Li, J.-Z., Wang, X.-F., et al. 2012, Research in Astronomy and Astrophysics, 12, 1585

Hunter, D. J., Valenti, S., Kotak, R., et al. 2009, A\&A, 508, 371

Im, M., Choi, C., Lee, S.-Y., et al. 2017, The Astronomer's Telegram, 10481 
Iwamoto, K., Mazzali, P. A., Nomoto, K., et al. 1998, Nature, 395, 672

Iwamoto, K., Nakamura, T., Nomoto, K., et al. 2000, ApJ, 534, 660

Kaplan, K. F., Jogee, S., Kewley, L., et al. 2016, Monthly Notices of the Royal Astronomical Society, 462, 1642

Kasen, D., \& Bildsten, L. 2010, ApJ, 717, 245

Kilpatrick, C. D., Takaro, T., Foley, R. J., et al. 2018, MNRAS, 480, 2072

Kotulla, R., Fritze, U., Weilbacher, P., \& Anders, P. 2009, MNRAS, 396, 462

Li, X., Hjorth, J., \& Wojtak, R. 2014, ApJL, 796, L4

Liu, Y.-Q., Modjaz, M., Bianco, F. B., \& Graur, O. 2016, ApJ, 827, 90

Lyman, J. D., Bersier, D., James, P. A., et al. 2016, MNRAS, 457, 328

Magnier, E. A., Schlafly, E. F., Finkbeiner, D. P., et al. 2016, ArXiv e-prints, arXiv:1612.05242

Maund, J. R., Smartt, S. J., Kudritzki, R. P., Podsiadlowski, P., \& Gilmore, G. F. 2004, Nature, 427, 129

Mazzali, P. A., Valenti, S., Della Valle, M., et al. 2008, Science, 437, 1185

Meynet, G., \& Maeder, A. 2003, Astronomy \& Astrophysics, 404, 975

Modjaz, M., Kewley, L., Bloom, J. S., et al. 2011, ApJL, 731, L4

Modjaz, M., Kewley, L., Kirshner, R. P., et al. 2008, AJ, 135,1136

Modjaz, M., Li, W., Butler, N., et al. 2009, The Astronomical Journal, 702, 226

Modjaz, M., Blondin, S., Kirshner, R. P., et al. 2014, The Astronomical Journal, 147, 99

Nakamura, T., Mazzali, P. A., Nomoto, K., \& Iwamoto, K. 2001, ApJ, 550, 991

Nicholl, M., Guillochon, J., \& Berger, E. 2017, ApJ, 850, 55

Ofek, E. O., Sullivan, M., Shaviv, N. J., et al. 2014, ApJ, 789,104

Patat, F., Cappellaro, E., Danziger, J., et al. 2001, Astrophysical Journal, 555, 900

Paxton, B., Marchant, P., Schwab, J., et al. 2015, ApJS, 220, 15

Piro, A. L. 2015, Astrophysical Journal, 808, L51

Piro, A. L., \& Nakar, E. 2013, ApJ, 769, 67

Podsiadlowski, P., Hsu, J. J. L., Joss, P. C., \& Ross, R. R. 1993, Nature, 364, 509

Poole, T. S., Breeveld, A. A., Page, M. J., et al. 2008, MNRAS, 383, 627

Poznanski, D., Butler, N., Filippenko, A. V., et al. 2009, ApJ, 694, 1067
Prentice, S. J., \& Mazzali, P. A. 2017, MNRAS, 469, 2672

Prentice, S. J., Ashall, C., Mazzali, P. A., et al. 2018, MNRAS, 478, 4162

Richmond, M. W., van Dyk, S. D., Ho, W., et al. 1996, The Astronomical Journal, 111, 327

Rodríguez, Ó., Clocchiatti, A., \& Hamuy, M. 2014, AJ, 148, 107

Roming, P. W. A., Kennedy, T. E., Mason, K. O., et al. 2005, SSRv, 120, 95

Sander, A., Hamann, W.-R., \& Todt, H. 2012, Astronomy \& Astrophysics, 540, A144

Schlafly, E. F., \& Finkbeiner, D. P. 2011, The Astrophysical Journal, 737, 103

Smartt, S. J. 2015, Publications of the Astronomical Society of Australia, 32, 16

Smartt, S. J., Eldridge, J. J., Crockett, R. M., \& Maund, J. R. 2009, MNRAS, 395, 1409

Smith, L. F., \& Maeder, A. 1991, Astronomy \& Astrophysics, 241, 77

Smith, N. 2014, ARA\&A, 52, 487

Stanway, E. R., \& Eldridge, J. J. 2018, MNRAS, 479, 75

Taddia, F., Fremling, C., Sollerman, J., et al. 2016, A\&A, 592, A89

Taubenberger, S., Pastorello, A., Mazzali, P. A., et al. 2006, MNRAS, 371, 1459

Thomas, R. C., Nugent, P. E., \& Meza, J. C. 2011, PASP, 123,237

Tody, D. 1986, in Proc. SPIE, Vol. 627, Instrumentation in astronomy VI, ed. D. L. Crawford, 733

Tody, D. 1993, in Astronomical Society of the Pacific Conference Series, Vol. 52, Astronomical Data Analysis Software and Systems II, ed. R. J. Hanisch, R. J. V. Brissenden, \& J. Barnes, 173

Tonry, J. L., Denneau, L., Heinze, A. N., et al. 2018, PASP, 130, 064505

Tully, R. B. 1988, Nearby galaxies catalog

Turatto, M., Benetti, S., \& Cappellaro, E. 2003, in From Twilight to Highlight: The Physics of Supernovae, 200

Valenti, S., Elias-Rosa, N., Taubenberger, S., et al. 2008, The Astrophysical Journal Letters, 673, L155

van der Hucht, K. A. 2006, A\&A, 458, 453

Van Dyk, S. D., Zheng, W., Brink, T. G., et al. 2018, ApJ, 860,90

Wang, L.-J., Wang, S. Q., Dai, Z. G., et al. 2016, The Astrophysical Journal, 821, 22

Wang, L. J., Yu, H., Liu, L. D., et al. 2017, The Astrophysical Journal, 837, 128

Waxman, E., \& Katz, B. 2016, ArXiv e-prints, arXiv:1607.01293 
Waxman, E., Mésázros, P., \& Campana, S. 2007, The Astrophysical Journal, 667, 351

Williams, P. M., Marchenko, S. V., Marston, A. P., et al. 2009, MNRAS, 395, 1749

Xiang, D., Song, H., Wang, X., et al. 2017, The Astronomer's Telegram, 10434

Yamanaka, M., Nakaoka, T., Tanaka, M., et al. 2017, ApJ, 837, 1
Yaron, O., Perley, D. A., Gal-Yam, A., et al. 2017, Nature Physics, 13, 510

Yokoo, T., Arimoto, J., Matsumoto, K., Takahashi, A., \& Sadakane, K. 1994, PASJ, 46, L191

Yoon, S.-C., Chun, W., Tolstov, A., Blinnikov, S., \& Dessart, L. 2018, ArXiv e-prints, arXiv:1810.03108 
Table 7. Optical BVRI photometry of SN 2017ein.

\begin{tabular}{|c|c|c|c|c|c|}
\hline MJD & $B$ & V & $R$ & $I$ & Telescope \\
\hline 57904.605 & $16.438 \pm 0.026$ & $15.885 \pm 0.013$ & $15.571 \pm 0.030$ & $15.245 \pm 0.017$ & TNT \\
\hline 57905.604 & $16.270 \pm 0.044$ & $15.715 \pm 0.022$ & $15.390 \pm 0.030$ & $15.076 \pm 0.020$ & TNT \\
\hline 57906.598 & $16.083 \pm 0.026$ & $15.506 \pm 0.016$ & $15.208 \pm 0.032$ & $14.912 \pm 0.021$ & TNT \\
\hline 57907.641 & $\ldots$ & $\ldots$ & $15.165 \pm 0.016$ & $14.835 \pm 0.032$ & TNT \\
\hline 57908.574 & $15.929 \pm 0.028$ & $15.347 \pm 0.014$ & $15.057 \pm 0.029$ & $14.735 \pm 0.015$ & TNT \\
\hline 57911.612 & $15.975 \pm 0.019$ & $15.221 \pm 0.011$ & $14.881 \pm 0.028$ & $14.531 \pm 0.015$ & TNT \\
\hline 57912.592 & $16.038 \pm 0.022$ & $15.209 \pm 0.012$ & $14.852 \pm 0.030$ & $14.496 \pm 0.014$ & TNT \\
\hline 57917.607 & $16.483 \pm 0.030$ & $15.312 \pm 0.012$ & $14.851 \pm 0.029$ & $14.457 \pm 0.016$ & TNT \\
\hline 57919.573 & $16.644 \pm 0.029$ & $15.436 \pm 0.016$ & $14.928 \pm 0.033$ & $14.490 \pm 0.016$ & TNT \\
\hline 57921.607 & $16.910 \pm 0.055$ & $15.542 \pm 0.019$ & $15.034 \pm 0.032$ & $14.547 \pm 0.026$ & TNT \\
\hline 57930.549 & $17.678 \pm 0.035$ & $16.280 \pm 0.018$ & $15.626 \pm 0.037$ & $14.982 \pm 0.020$ & TNT \\
\hline 57932.560 & $\ldots$ & $\ldots$ & $15.773 \pm 0.079$ & $\ldots$ & TNT \\
\hline 57933.566 & $\ldots$ & $16.591 \pm 0.134$ & $15.855 \pm 0.053$ & $15.258 \pm 0.071$ & TNT \\
\hline 57936.713 & $18.190 \pm 0.021$ & $16.790 \pm 0.016$ & $16.084 \pm 0.012$ & $15.338 \pm 0.013$ & $\mathrm{AZT}$ \\
\hline 57937.712 & $18.184 \pm 0.026$ & $16.804 \pm 0.016$ & $16.127 \pm 0.008$ & $\ldots$ & $\mathrm{AZT}$ \\
\hline 57938.726 & $18.150 \pm 0.029$ & $16.831 \pm 0.017$ & $16.182 \pm 0.008$ & $15.460 \pm 0.013$ & $\mathrm{AZT}$ \\
\hline 57940.694 & $18.334 \pm 0.047$ & $16.921 \pm 0.020$ & $16.257 \pm 0.010$ & $15.512 \pm 0.019$ & AZT \\
\hline 57941.711 & $18.344 \pm 0.021$ & $16.958 \pm 0.017$ & $16.287 \pm 0.008$ & $15.557 \pm 0.014$ & $\mathrm{AZT}$ \\
\hline 57942.723 & $18.325 \pm 0.040$ & $16.944 \pm 0.023$ & $16.330 \pm 0.010$ & $15.562 \pm 0.013$ & AZT \\
\hline 57945.685 & $18.121 \pm 0.116$ & $17.044 \pm 0.051$ & $16.365 \pm 0.011$ & $15.598 \pm 0.013$ & AZT \\
\hline 57946.721 & $18.606 \pm 0.110$ & $17.001 \pm 0.022$ & $16.364 \pm 0.011$ & $15.600 \pm 0.013$ & $\mathrm{AZT}$ \\
\hline 57949.687 & $18.335 \pm 0.072$ & $17.152 \pm 0.021$ & $16.488 \pm 0.013$ & $15.683 \pm 0.021$ & $\mathrm{AZT}$ \\
\hline 57950.695 & $18.556 \pm 0.028$ & $17.093 \pm 0.017$ & $16.503 \pm 0.011$ & $15.678 \pm 0.014$ & $\mathrm{AZT}$ \\
\hline 57951.695 & $18.431 \pm 0.018$ & $17.171 \pm 0.015$ & $16.551 \pm 0.008$ & $15.735 \pm 0.022$ & $\mathrm{AZT}$ \\
\hline 57952.684 & $18.500 \pm 0.094$ & $17.210 \pm 0.020$ & $16.544 \pm 0.012$ & $15.732 \pm 0.019$ & $\mathrm{AZT}$ \\
\hline 57953.678 & $18.524 \pm 0.030$ & $17.227 \pm 0.024$ & $16.609 \pm 0.016$ & $15.761 \pm 0.015$ & AZT \\
\hline 57954.686 & $18.600 \pm 0.018$ & $17.239 \pm 0.019$ & $16.621 \pm 0.015$ & $15.788 \pm 0.018$ & $\mathrm{AZT}$ \\
\hline 57955.686 & $18.684 \pm 0.102$ & $17.277 \pm 0.016$ & $16.634 \pm 0.010$ & $15.804 \pm 0.016$ & AZT \\
\hline 57956.693 & $18.800 \pm 0.120$ & $17.269 \pm 0.026$ & $16.615 \pm 0.017$ & $15.796 \pm 0.017$ & AZT \\
\hline 57957.674 & $18.793 \pm 0.109$ & $17.324 \pm 0.033$ & $16.686 \pm 0.020$ & $15.881 \pm 0.014$ & AZT \\
\hline 57958.683 & $18.715 \pm 0.049$ & $17.338 \pm 0.024$ & $16.715 \pm 0.009$ & $15.888 \pm 0.013$ & AZT \\
\hline 57960.683 & $18.642 \pm 0.166$ & $17.296 \pm 0.017$ & $16.696 \pm 0.022$ & $15.852 \pm 0.013$ & AZT \\
\hline 57963.670 & $18.460 \pm 0.039$ & $17.449 \pm 0.046$ & $16.786 \pm 0.011$ & $15.908 \pm 0.016$ & $\mathrm{AZT}$ \\
\hline 57964.667 & $18.615 \pm 0.150$ & $17.367 \pm 0.020$ & $16.820 \pm 0.008$ & $15.921 \pm 0.017$ & $\mathrm{AZT}$ \\
\hline 57965.667 & $18.820 \pm 0.028$ & $17.410 \pm 0.034$ & $16.827 \pm 0.015$ & $15.969 \pm 0.016$ & $\mathrm{AZT}$ \\
\hline 58107.022 & $21.858 \pm 1.412$ & $20.151 \pm 0.098$ & $19.639 \pm 0.045$ & $19.456 \pm 0.239$ & AZT \\
\hline 58124.882 & $21.636 \pm 1.494$ & $22.635 \pm 1.440$ & $19.852 \pm 0.108$ & $21.230 \pm 1.004$ & AZT \\
\hline 58133.075 & $20.097 \pm 1.340$ & $19.113 \pm 0.391$ & $19.432 \pm 0.128$ & $19.826 \pm 0.242$ & AZT \\
\hline 58139.040 & $20.039 \pm 0.322$ & $22.644 \pm 1.036$ & $21.158 \pm 0.104$ & $20.422 \pm 0.098$ & $\mathrm{AZT}$ \\
\hline
\end{tabular}

Table 7 continued 
XiAng ET AL.

Table 7 (continued)

\begin{tabular}{cccccc}
\hline \hline MJD & $B$ & $V$ & $R$ & $I$ & Telescope \\
\hline 58139.962 & $20.581 \pm 0.195$ & $\ldots$ & $21.487 \pm 1.077$ & $20.267 \pm 0.519$ & AZT \\
58140.954 & $\ldots$ & $21.599 \pm 0.905$ & $21.372 \pm 1.064$ & $20.900 \pm 0.605$ & AZT \\
58143.896 & $19.676 \pm 0.611$ & $23.596 \pm 1.241$ & $20.507 \pm 0.286$ & $20.018 \pm 0.346$ & AZT \\
58150.919 & $20.158 \pm 1.441$ & $21.834 \pm 0.605$ & $19.399 \pm 0.246$ & $21.078 \pm 0.933$ & AZT \\
58152.898 & $\ldots$ & $21.061 \pm 0.673$ & $20.245 \pm 0.280$ & $\ldots$ & AZT \\
58154.958 & $21.784 \pm 0.727$ & $21.371 \pm 0.196$ & $20.674 \pm 0.194$ & $\ldots$ & AZT \\
58156.966 & $\ldots$ & $20.820 \pm 0.124$ & $20.259 \pm 0.082$ & $20.318 \pm 0.293$ & AZT \\
58167.949 & $20.121 \pm 0.756$ & $20.633 \pm 0.149$ & $21.456 \pm 0.336$ & $19.844 \pm 0.425$ & AZT \\
58170.917 & $19.208 \pm 0.393$ & $24.017 \pm 1.374$ & $20.768 \pm 0.668$ & $21.959 \pm 0.550$ & AZT \\
58171.904 & $20.520 \pm 0.840$ & $21.682 \pm 0.310$ & $21.252 \pm 0.136$ & $20.347 \pm 0.227$ & AZT \\
\hline
\end{tabular}

Table 8. ATLAS photometry of SN 2017ein

\begin{tabular}{cccc|ccccc}
\hline \hline MJD & AB mag & err & \multicolumn{1}{l}{ Filter } & MJD & AB mag & err & Filter \\
\hline 57869.506 & $>20.06$ & 1.35 & c & 57907.281 & 15.17 & 0.01 & o \\
57869.520 & $>20.01$ & 0.53 & c & 57907.296 & 15.16 & 0.01 & o \\
57869.535 & $>19.87$ & 2.18 & c & 57908.269 & 15.29 & 0.11 & o \\
57869.550 & $>19.67$ & 0.00 & c & 57908.284 & 15.25 & 0.05 & o \\
57870.425 & $>20.13$ & 0.00 & c & 57908.294 & 15.17 & 0.04 & o \\
57870.437 & $>20.19$ & 0.00 & c & 57911.261 & 14.84 & 0.01 & o \\
57870.444 & $>20.21$ & 0.45 & c & 57911.284 & 14.84 & 0.01 & o \\
57870.459 & $>20.11$ & 0.00 & c & 57911.300 & 14.84 & 0.01 & o \\
57870.482 & $>20.12$ & 0.56 & c & 57912.260 & 14.83 & 0.01 & o \\
57882.354 & $>19.34$ & 0.61 & o & 57912.262 & 14.84 & 0.01 & o \\
57882.380 & $>19.52$ & 0.00 & o & 57912.274 & 14.80 & 0.01 & o \\
57882.391 & $>19.54$ & 0.43 & o & 57912.291 & 14.79 & 0.01 & o \\
57882.415 & $>19.44$ & 2.92 & o & 57912.291 & 14.80 & 0.01 & o \\
57887.280 & $>19.83$ & 0.00 & o & 57912.304 & 14.85 & 0.01 & o \\
57887.296 & $>19.80$ & 0.73 & o & 57912.305 & 14.83 & 0.01 & o \\
57887.307 & $>19.47$ & 0.00 & o & 57914.269 & 14.93 & 0.01 & o \\
57887.314 & $>19.64$ & 0.00 & o & 57914.271 & 14.93 & 0.01 & o \\
57887.320 & $>19.33$ & 0.00 & o & 57914.290 & 14.88 & 0.01 & o \\
57887.335 & $>19.59$ & 0.97 & o & 57914.299 & 14.90 & 0.01 & o \\
57887.339 & $>19.64$ & 0.68 & o & 57914.302 & 14.92 & 0.01 & o \\
57887.354 & $>19.62$ & 1.02 & o & 57914.304 & 14.90 & 0.01 & o \\
57892.322 & $>19.95$ & 0.81 & o & 57914.318 & 14.94 & 0.01 & o \\
57892.324 & $>19.72$ & 0.00 & o & 57914.327 & 14.89 & 0.01 & o \\
57892.337 & $>19.75$ & 1.85 & o & 57914.335 & 14.91 & 0.01 & o \\
57894.333 & $>20.24$ & 0.00 & c & 57915.310 & 14.83 & 0.01 & o \\
\hline & & & & & & &
\end{tabular}

Table 8 continued 
A Type IC Supernova SN 2017ein

Table 8 (continued)

\begin{tabular}{cccc|cccc}
\hline \hline MJD & AB mag & err & \multicolumn{1}{l}{ Filter } & MJD & AB mag & err & Filter \\
\hline 57894.356 & $>20.02$ & 0.00 & c & 57915.333 & 14.81 & 0.01 & o \\
57894.370 & $>20.09$ & 0.97 & c & 57915.355 & 14.82 & 0.01 & o \\
57894.390 & $>20.07$ & 1.40 & c & 57918.269 & 14.86 & 0.01 & o \\
57894.404 & $>20.11$ & 0.00 & c & 57918.280 & 14.87 & 0.01 & o \\
57896.252 & $>20.04$ & 1.41 & o & 57922.260 & 15.00 & 0.01 & o \\
57896.267 & $>20.07$ & 0.00 & o & 57922.270 & 15.03 & 0.02 & o \\
57896.283 & $>19.61$ & 0.66 & o & 57925.250 & 15.15 & 0.01 & o \\
57898.336 & 18.43 & 0.10 & c & 57925.260 & 15.16 & 0.01 & o \\
57898.354 & 18.69 & 0.14 & c & 57925.278 & 15.17 & 0.01 & o \\
57898.362 & 18.53 & 0.11 & c & 57925.283 & 15.15 & 0.01 & o \\
57898.384 & 18.35 & 0.10 & c & 57927.278 & 15.38 & 0.01 & o \\
57898.390 & 18.25 & 0.10 & c & 57927.287 & 15.40 & 0.01 & o \\
57900.255 & 16.98 & 0.05 & o & 57929.259 & 15.55 & 0.01 & o \\
57900.262 & 16.92 & 0.04 & o & 57929.262 & 15.53 & 0.01 & o \\
57900.267 & 17.07 & 0.05 & o & 57929.277 & 15.52 & 0.01 & o \\
57900.280 & 16.83 & 0.04 & o & 57929.286 & 15.52 & 0.01 & o \\
57900.284 & 17.02 & 0.05 & o & 57933.259 & 15.75 & 0.01 & o \\
57900.313 & 17.05 & 0.05 & o & 57933.262 & 15.80 & 0.02 & o \\
57903.256 & 15.96 & 0.02 & o & 57933.276 & 15.74 & 0.01 & o \\
57903.270 & 15.88 & 0.02 & o & 57933.287 & 15.79 & 0.02 & o \\
57903.285 & 15.81 & 0.02 & o & 57937.258 & 16.00 & 0.02 & o \\
57903.299 & 15.81 & 0.02 & o & 57937.263 & 16.01 & 0.02 & o \\
57907.259 & 15.17 & 0.01 & o & 57937.273 & 16.04 & 0.02 & o \\
57907.264 & 15.18 & 0.01 & o & 57937.288 & 16.03 & 0.02 & o \\
\hline & & & & & & &
\end{tabular}


Table 9. Swift-UVOT photometry of SN 2017ein.

\begin{tabular}{ccccc}
\hline \hline MJD & uvw 1 & $u$ & $b$ & $v$ \\
\hline 57900.510 & $\ldots$ & $17.619 \pm 0.213$ & $17.943 \pm 0.182$ & $17.133 \pm 0.181$ \\
57911.922 & $17.674 \pm 0.176$ & $15.980 \pm 0.084$ & $15.966 \pm 0.069$ & $15.228 \pm 0.070$ \\
57915.465 & $17.713 \pm 0.189$ & $16.812 \pm 0.123$ & $16.188 \pm 0.075$ & $15.221 \pm 0.072$ \\
57919.892 & $\ldots$ & $17.473 \pm 0.171$ & $16.643 \pm 0.084$ & $15.401 \pm 0.074$ \\
57923.103 & $\ldots$ & $18.238 \pm 0.288$ & $17.094 \pm 0.099$ & $15.737 \pm 0.080$ \\
57927.849 & $\ldots$ & $18.500 \pm 0.368$ & $17.775 \pm 0.149$ & $16.292 \pm 0.105$ \\
57933.992 & $\ldots$ & $\ldots$ & $18.114 \pm 0.224$ & $16.764 \pm 0.162$ \\
57937.877 & $\ldots$ & $\ldots$ & $18.518 \pm 0.256$ & $16.796 \pm 0.138$ \\
57942.460 & $\ldots$ & $\ldots$ & $18.409 \pm 0.240$ & $16.928 \pm 0.154$ \\
57945.483 & $\ldots$ & $\ldots$ & $18.424 \pm 0.250$ & $17.078 \pm 0.174$ \\
57949.869 & $\ldots$ & $\ldots$ & $18.382 \pm 0.346$ & $16.699 \pm 0.196$ \\
57953.332 & $\ldots$ & $\ldots$ & $18.350 \pm 0.248$ & $16.950 \pm 0.173$ \\
57955.423 & $\ldots$ & $\ldots$ & $18.253 \pm 0.205$ & $17.057 \pm 0.161$ \\
57960.206 & $\ldots$ & $\ldots$ & $18.568 \pm 0.285$ & $17.015 \pm 0.175$ \\
\hline
\end{tabular}

